\title{
On the flow characteristics in different carotid arteries ${ }^{\circledR}$
}

Cite as: Phys. Fluids 32, 101902 (2020); https://doi.org/10.1063/5.0022092

Submitted: 17 July 2020 . Accepted: 19 September 2020 . Published Online: 05 October 2020

(D) Yu Chen (陈与), (D) Xiaolei Yang (杨晓雷), Andrew John Iskander, and Ping Wang (王萍)

\section{COLLECTIONS}

EP This paper was selected as an Editor's Pick

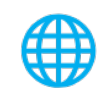

\section{ARTICLES YOU MAY BE INTERESTED IN}

Elasto-hydrodynamic interaction of two swimming spermatozoa

Physics of Fluids 32, 101901 (2020); https://doi.org/10.1063/5.0022107

A mathematical framework for estimating risk of airborne transmission of COVID-19 with application to face mask use and social distancing

Physics of Fluids 32, 101903 (2020); https://doi.org/10.1063/5.0025476

Reducing chances of COVID-19 infection by a cough cloud in a closed space

Physics of Fluids 32, 101704 (2020); https://doi.org/10.1063/5.0029186

\section{Physics of Fluids GALLERY OF GOVERS}




\title{
On the flow characteristics in different carotid arteries
}

\author{
Cite as: Phys. Fluids 32, 101902 (2020); doi: 10.1063/5.0022092 \\ Submitted: 17 July 2020 - Accepted: 19 September 2020 • \\ Published Online: 5 October 2020
}

Yu Chen (陈与), ${ }^{1,2}$ (D) Xiaolei Yang (杨晓雷), ${ }^{1,3, a)}$ (D) Andrew John Iskander, ${ }^{4}$ and Ping Wang (王萍) ${ }^{2}$

\begin{abstract}
AFFILIATIONS
${ }^{1}$ State Key Laboratory of Nonlinear Mechanics, Institute of Mechanics, Chinese Academy of Sciences, Beijing 100190, China

${ }^{2}$ Department of Mechanics, Lanzhou University, Lanzhou 730000, China

${ }^{3}$ School of Engineering Sciences, University of Chinese Academy of Sciences, Beijing 100049, China

4Department of Anesthesiology, Westchester Medical Center, 100 Woods Road, Valhalla, New York 10595, USA
\end{abstract}

a) Author to whom correspondence should be addressed: xyang@imech.ac.cn

\begin{abstract}
Hemodynamics plays an important role in the cause of atherosclerotic disease. In this work, we simulated the flow fields in six human carotids using the sharp-interface immersed boundary method. We compare the axial velocity, the secondary flow, the vortex structures, the area with reversed flows, and the standard deviation of velocity fluctuations for different carotids. The computed results show similarities among different carotids for the overall pattern of different flow characteristics. More importantly, differences are observed in terms of the details of the area of the reversed flow region, the size and shape of the region dominated by reversed flows, and the length of time with reversed flow or dominated by secondary flows, which are strongly correlated with the formation of plaques. Further analysis shows that patient-specific features of the carotid geometry, e.g., the flare and proximal curvature of the carotid, have significant effects on these flow characteristics. This suggests that a patient-specific program is needed for the prevention and treatment of atherosclerosis.
\end{abstract}

Published under license by AIP Publishing. https://doi.org/10.1063/5.0022092

\section{INTRODUCTION}

Atherosclerosis is one of the common diseases of the elderly, which is extremely harmful to people's health. Studies have shown that hemodynamics is closely related to atherosclerosis diseases. ${ }^{1}$ For instance, it was suggested by Caro et al. ${ }^{2}$ that the initial portions of atherosclerosis start from the region with disturbed flow and low wall shear stress (WSS). Ku et al. ${ }^{1}$ further suggested that the high wall shear stress gradient (WSSG) and high oscillatory shear index (OSI) are inseparable with cardiovascular diseases.

However, the correlation between wall shear stress and the pathogenesis of atherosclerosis is still not clear yet. Fry ${ }^{3}$ first speculated that high wall shear can damage endothelial cells to cause atherosclerosis through the animal vivo experiments. In contrast to Fry's conclusion, Caro et al., ${ }^{2}$ Friedman et al., ${ }^{4}$ and $\mathrm{Ku}$ et al. ${ }^{1}$ observed that the low wall shear regions are the concentrated areas of atherosclerotic plaques. Although there is no clear explanation for the relationship between WSS and atherosclerosis, most researchers are convicted by the low WSS mechanism. ${ }^{5}$ However, some studies also suggested that the association is not so strong. ${ }^{6}$ The high WSS mechanism is supported by animal anatomy experiments, but vitro measurements demonstrated that the low WSS can cause atherosclerosis. ' In recent studies, researchers attempted to probe into the vector property of wall shear stress to find the association between hemodynamic factors and atherosclerosis. For instance, Peiffer et al..$^{10}$ defined a cross-transverse wall shear stress, which is the component of time-averaged wall shear stress perpendicular to the mean flow direction, to characterize the multi-directional property of the shear stress vector. Qiu and Tarbell ${ }^{11}$ suggested that the stress phase angle (SPA), which is defined as the temporal phase angle between WSS and circumferential stress (CS), can be another factor that affects the pathogenesis of atherosclerosis.

It is noticed that most studies have been focused on the wall shear stress ${ }^{12,13}$ or other indicators ${ }^{14}$ [such as oscillatory shear index (OSI) and relative residence time, which are functions of the wall shear stress] for identifying the region of vascular wall dysfunction without looking into the flow structure within the artery. For instance, Bevan et al. ${ }^{15}$ investigated the wall shear stress 
distribution in a patient-specific carotid. Pinto et al. ${ }^{16}$ investigated the wall shear stress distribution for a carotid bifurcation with stenosis. Yao et al. ${ }^{17}$ examined the correlation between the time-averaged wall shear stress and the oscillatory shear index with the location of neointimal hyperplasia. Nagargoje and Gupta ${ }^{18}$ studied the effect of the size and position of carotid sinus on the distribution of the wall shear stress and streamwise velocity for an ideal carotid.

Studies on the effects of carotid geometry on flow structures are limited to ideal carotids or simplified problems. For instance, Rindt and Steenhoven ${ }^{19}$ investigated the pulsatile flow in an ideal carotid artery and found C-shaped axial velocity pattern and Deantype vortices at the deceleration phase and at the end systole phase. Plesniak and Bulusu ${ }^{20}$ investigated the pattern of the secondary flow in a curved channel using particle image velocimetry (PIV) experiments. Najjari and Plesniak ${ }^{21,22}$ probed into the secondary flow vortical structures in an ideal curved vessel. Janiga ${ }^{23}$ studied the temporal and spatial variations of blood flow in cerebral aneurysms using proper orthogonal decomposition. Studies on the flow structures in the stenosed artery have also been carried out, ${ }^{24,25}$ which are often featured by jet flows, increased turbulence intensity, and increased wall shear stress. For instance, Lancellotti et al. ${ }^{26}$ investigated the velocity and vorticity pattern of a real stenotic carotid. Kang et al. ${ }^{2}$ compared the axial velocity, axial vorticity, and rate of energy dissipation from a stenosed carotid bifurcation with those from a normal carotid bifurcation.

The distributions of the wall shear stress are highly correlated with the flow structures within the artery, for instance, the regions of low WSS and high OSI are influenced by the reflux velocity. However, for arteries with complex geometries, such as the carotid artery with bifurcations and curvatures, it is difficult to give a clear pathological explanation on why the lesion regions are mainly located at curved branch and bifurcations because of the flow complexity. Therefore, it is necessary to carefully study the flow field within patient-specific carotid arteries for a better understanding of the effect of complex flow structures on the pathogenesis of atherosclerosis.

In this work, we numerically study the flow structures in six different carotid arteries, which were reconstructed by MRA (Magnetic Resonance Angiograms) technology. We employ direct numerical simulation with the immersed boundary method for representing the geometry of the carotids. Three key contributions from this work are highlighted as follows: (1) the temporal and spatial variations of flow structures in six patient-specific carotids are investigated; (2) effects of the carotid flare and proximal curvature on the reversed flow and the intensity of velocity variations are examined; and (3) the simulation results indicate that the carotid with high flare and low curvature is more susceptible to atherosclerosis, which is consistent with the literature. ${ }^{28}$

The rest of this paper is organized as follows: the employed numerical method is briefly described in Sec. II; then, the simulation results and discussion are presented in Secs. III and IV, respectively; at last, conclusions are drawn in Sec. V.

\section{METHODOLOGY}

\section{A. Numerical method}

In this study, the blood is assumed to be incompressible and Newtonian. The blood flow within the carotid is simulated using the
Virtual Flow Simulator (VFS) code. ${ }^{29}$ The governing equations are the continuity and the Navier-Stokes equations shown as follows:

$$
\begin{gathered}
\nabla \cdot \mathbf{u}=0, \\
\frac{\partial \mathbf{u}}{\partial t}+\mathbf{u} \cdot \nabla \mathbf{u}=-\frac{1}{\rho} \nabla \mathbf{p}+\frac{\mu}{\rho} \nabla^{2} \mathbf{u},
\end{gathered}
$$

where $\mathbf{u}$ is the velocity, $\mathbf{p}$ is the pressure, $\rho$ is the density of blood, and $\mu$ is the dynamic viscosity of blood. In this work, $\rho=1050 \mathrm{~kg} / \mathrm{m}^{3}$, and $\mu=0.0037 \mathrm{~N} \mathrm{~s} / \mathrm{m}^{2}$. The governing equations are discretized spatially using a second-order central differencing scheme and temporally using a second-order accurate fractional step method. The pressure Poisson equation is solved using an algebraic multigrid acceleration along with a generalized minimal residual solver. The momentum equation is solved using the matrix-free Newton-Krylov method.

The carotid geometry is represented using the sharp-interface curvilinear immersed boundary (CURVIB) method. ${ }^{30}$ In the CURVIB method, the blood flow is solved on a non-boundaryconforming Cartesian or curvilinear grid. The carotid geometry is discretized via an unstructured triangular mesh immersed into the background non-boundary conforming grid. In general, the grid nodes of the background grid do not coincide with the immersed carotid surface mesh such that the boundary conditions cannot be applied at the boundary directly. In the CURVIB method, the boundary conditions are applied at the IB (immersed boundary) nodes, which are located in the fluid with at least one neighbor in the solid. The velocities at the IB nodes are reconstructed from the values at the boundary and the surrounding fluid nodes using a linear interpolation in the wall-normal direction. The flow solver and the CURVIB code employed in the VFS code have been successfully applied to blood flow simulations. ${ }^{31-33}$

\section{B. Case setup}

In this work, we simulate six right carotid arteries, for which the source materials for generating the carotid geometry were obtained from neck magnetic resonance angiograms performed at the Stony Brook Medical Center from June to September of 2018 as part of an adult stroke protocol. ${ }^{34}$ These scans were selected and determined to be in compliance with the institutional review board approval. These images were then reviewed anonymously and determined to be without filling defect and cropped to include similar segments of the right carotid bifurcation. Six scans were selected, which met this criterion (five males and one female). Using the open source software 3D-Slicer 4.8.1 (www.slicer.org), the meshes were cropped and converted from DICOM to STL format to create the meshes. Figure 1 shows the 3D images of the six carotid arteries. The geometries of $\mathrm{C} 1$ and $\mathrm{C} 2$ are similar. Other than this, significant differences exist in the geometries of the carotid arteries from different individuals. It is noticed that the images are not plotted in the same scale. To quantitatively examine the differences among different carotids, we show in Table I the area of the three cross sections of the carotid (shown in Fig. 1). It is noticed that the areas of different sections are different for different carotids. The maximum section area exists at the I 2 or I3 section for all carotids. It is also observed that the change in the section area in the streamwise direction is small for $\mathrm{C} 1$ and $\mathrm{C} 2$, while it is very large for C4, C5, and C6. Especially, the area of cross section 

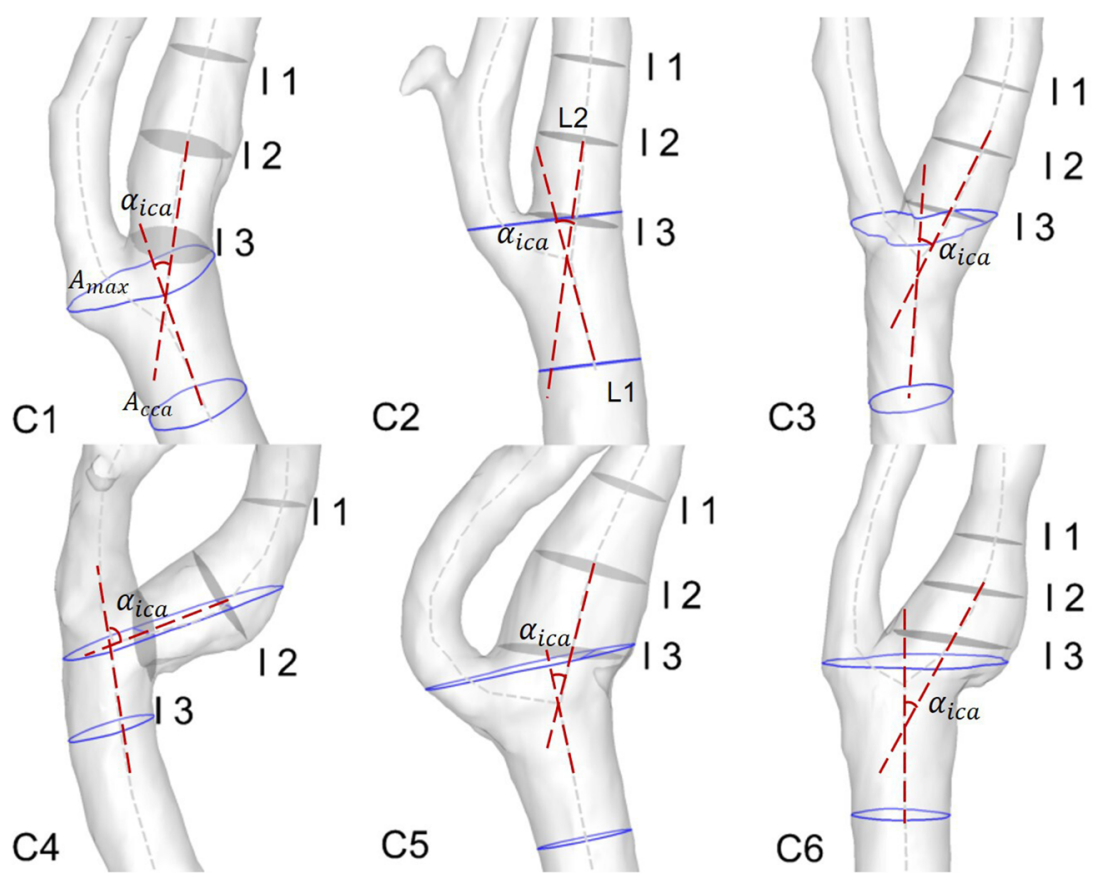

FIG. 1. The 3D geometric image of six carotid arteries. CCA: common carotid artery, ICA: internal carotid artery, and ECA: external carotid artery. In this image, 11,12 , and 13 show the three slices at different downstream locations in the carotid sinus, where we systematically investigate the flow characteristics. The areas of the cross sections with blue boundaries are employed for computing the flare. The proximal curvature is measured using the angle $\alpha_{\text {ica }}$. The values of the flare and $\alpha_{i c a}$ are listed in Table I.

I3 is approximately 2.7 times larger than I1 for C5. The flare of the common carotid artery (CCA) as approaching the bifurcation point and the proximal curvature are also quantified and listed in Table I, which have been used in the literature for assessing the geometric risk of different carotids for atherosclerosis. ${ }^{28}$ As illustrated in Fig. 1, the flare is defined as $A_{\max } / A_{c c a}$, where $A_{c c a}$ is the minimal area of the cross section (with its normal along the axial direction of CCA) in the considered part of CCA and $A_{\max }$ is the maximal area of the cross section (with its normal the same as the cross section for computing $A_{c c a}$ ) before the bifurcation point. The proximal curvature is measured using $\alpha_{i c a}$, which is the angle defined using the normal direction of cross section $A_{c c a}$ and the line connecting the centroids of $I 2$ and I3. It is noticed that either the internal carotid artery (ICA) or the CCA may deviate from the plane approximately defined using the ICA and external carotid artery (ECA) branches. This is the reason why the actual value of $\alpha_{i c a}$ is larger than it appears in Fig. 1 for

TABLE I. The cross-sectional area at three sections, the flare, and the curvature of the six carotids. As illustrated in Fig. 1, the flare and proximal curvature are defined as $A_{\max } / A_{c c a}$ and measured using $\alpha_{\text {ica }}$, respectively.

\begin{tabular}{lccccc}
\hline \hline Area $\left(\mathrm{mm}^{2}\right)$ & I1 & I2 & I3 & $\begin{array}{c}\text { Flare } \\
\left(A_{\text {max }} / A_{c c a}\right)\end{array}$ & $\begin{array}{c}\text { Curvature } \\
\left(\alpha_{\text {ica }}\right)\end{array}$ \\
\hline C1 & 19.38 & 27.79 & 25.36 & 1.51 & 32.17 \\
C2 & 16.09 & 21.43 & 27.71 & 1.36 & 10.58 \\
C3 & 20.95 & 30.00 & 33.36 & 1.98 & 34.22 \\
C4 & 19.58 & 49.80 & 50.81 & 3.67 & 80.71 \\
C5 & 28.00 & 56.73 & 75.71 & 3.43 & 31.17 \\
C6 & 17.64 & 35.58 & 51.47 & 2.50 & 25.08 \\
\hline \hline
\end{tabular}

carotid C1. It is noticed that the flare and the curvature are measured in a simple way in this work. A more systematic way can be found in the work by Lee et al. ${ }^{35}$ In this work, we will examine the flow fields on the three cross sections located in the carotid sinus, as shown in Fig. 1. Because of the complexity of the carotid geometry, it is hard to define exactly both the extent of the carotid sinus and the direction of the vascular axis. In this work, the three cross sections are manually selected in a way that they represent the typical locations relative to the carotid sinus with I1 in the downstream of the carotid sinus (near the end of sinus), I2 in the middle of the carotid sinus, and I3 in the upstream of the carotid sinus (near the bifurcation), respectively. In Appendix B, we examine the differences between flow structures on cross sections with slightly different orientations and locations and observe very similar flow patterns on different cross sections.

Figure 2 shows the grid employed in the C4 case. The surface of the carotid is discretized using unstructured triangular meshes. A uniformly distributed Cartesian grid with a grid spacing of $0.1 \mathrm{~mm}$ in all three directions is employed for all the cases. Figure 3 shows the flow rate during one cardiac cycle with several typical instants marked out, where the flow characteristics are examined in detail in this work. The pulsatile waveform employed in this work is prototypical for representing cardiac cycles' characteristics, ${ }^{36}$ which was obtained by Holdsworth et al. ${ }^{37}$ from the measurements of 48 volunteers and has been used in different studies. ${ }^{38}$ In the work by Ford et al., ${ }^{39}$ it was found that the shapes of the waveform from different individuals are similar although respective flow rates may be different. Considering patient-specific inflow is of interest in terms of its impact on flow structures and for patient-specific analysis. However, the inflow waveforms for the carotids considered in this work are not available. Probing into the effects of inflow waveform 


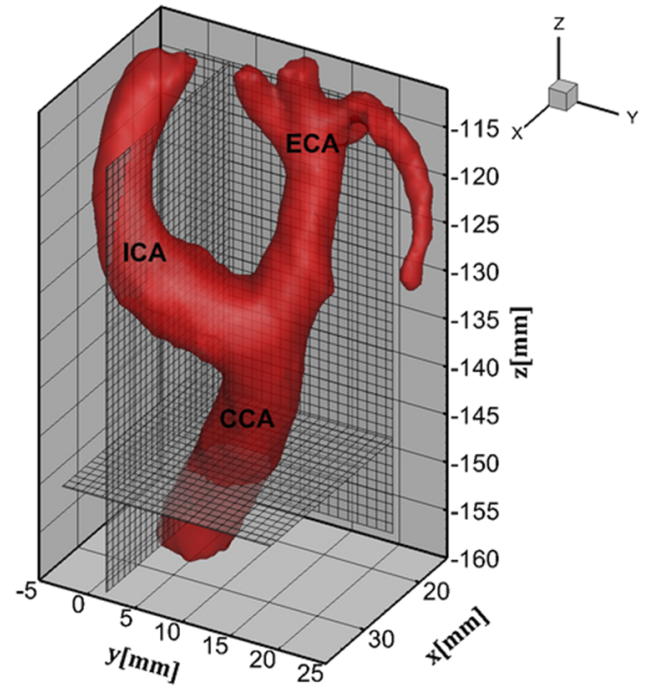

FIG. 2. The background Cartesian grid employed in the C4 case, in which every tenth grid line is displayed. The grid is uniform in all three directions with a grid spacing of $0.1 \mathrm{~mm}$.

will require series of simulations and can be carried out in the future work. The velocity at the inlet plane of the CCA is approximated by a parabolic distribution with its flow rate given in Fig. 3 . The flow rate at the outlet of the ECA is fixed at $26 \%$ of the total flow rate. Specifically, the velocity component normal to the outlet plane is specified as

$$
u_{N_{x}}=u_{N_{x}-1}+\frac{\alpha Q_{\text {in }}-Q_{N_{x}-1}}{A_{N_{x}}},
$$

where $N_{x}, N_{x}-1$ denote the outlet plane and the plane next to the outlet, respectively, $Q_{i n}$ is the flow rate at the inlet, $Q_{N_{x}-1}$ is the flow rate at the plane next to the outlet, $A_{N_{x}}$ is the area of the area of the outlet plane, and $\alpha=0.26$ is the ratio of the flow rate at the outlet of the ECA to the flow rate at the inlet of the CCA with its value determined based on Marshall et al.'s measurements of nine healthy volunteers. ${ }^{6}$ The other two components of the velocity at the outlet of ECA are specified using the Neumann boundary

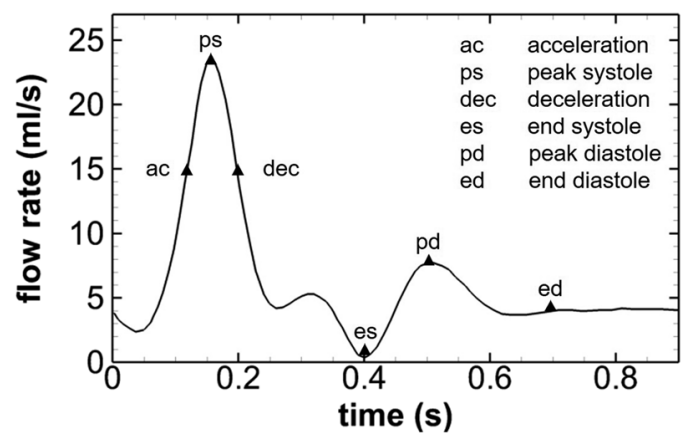

FIG. 3. The waveform of the pulsatile flow rate applied at the inlet of the CCA of the carotid artery.
TABLE II. The minimum and maximum time step in the simulations of the six carotids with the CFL number fixed at 0.8 .

\begin{tabular}{lcccccc}
\hline \hline$\Delta t\left(10^{-3} \mathrm{~s}\right)$ & $\mathrm{C} 1$ & $\mathrm{C} 2$ & $\mathrm{C} 3$ & $\mathrm{C} 4$ & $\mathrm{C} 5$ & C6 \\
\hline$\Delta t_{\min }$ & 0.0030 & 0.0450 & 0.0410 & 0.0520 & 0.0335 & 0.0060 \\
$\Delta t_{\max }$ & 0.6790 & 0.5490 & 0.4830 & 0.8010 & 0.6570 & 0.4990 \\
\hline
\end{tabular}

condition. At the outlet of ICA, the Neumann boundary condition is applied to all three velocity components. The vascular wall is considered to be rigid with no slip boundary conditions applied. In all case, the Courant-Friedrichs-Lewy (CFL) number is fixed at 0.8 with the minimum and maximum time step shown in Table II. For all cases, we run the simulation for two cardiac cycles and analyze the flow field in the second cardiac cycle. One case takes approximately $10 \mathrm{~h}$ using $320 \mathrm{cpu}$ cores.

\section{RESULTS}

\section{A. Qualitative analysis of flow characteristics}

In this work, we focus on the flow structures in the carotid sinus, where atherosclerotic plaques tend to develop and concentrate. Specifically, we analyze in detail the axial velocity, secondary flows, the secondary flow ratio (SFR), and vortex structures at particular moments of the cardiac cycle (Fig. 3), i.e., the acceleration (ac) instant, the peak of systole (ps) instant, the deceleration (dec) instant, the end of systole (es) instant, the peak of diastole (pd) instant, and the end of diastole (ed) instant, in which the secondary flow ratio $(S F R)$ is defined as

$$
S F R=\frac{\left|V_{S F}\right|}{|V|+\varepsilon}
$$

where $V_{S F}$ is the velocity magnitude of the secondary flow, $V$ is the velocity magnitude, and $\varepsilon$ is a small positive number to avoid the problem of division by zero.

We show the contours of axial velocity of blood flow in different carotids in Fig. 4. During the acceleration phase (from ac to ps instants), the pattern of the axial speed distribution composing of two regions, i.e., the high velocity region and the low speed region, is relatively simple. The relative locations of the two regions barely change from the ac instant to the ps instant, while the relative magnitudes significantly increase as a result of the increase in the incoming flow rate. All the six different carotids show the two regions of high speed and low speed but with different locations and shapes. During the deceleration phase, on the other hand, the distributions of the axial velocity become fairly complex on the three sections. Reversed flow is observed for all the six carotids at I 2 and I 3 sections. The magnitude of the reversed velocity is quite different among different carotids, in which the reversed flows in the C1, C2, C5, and C6 carotids (with the axial velocity magnitude as high as $-0.2 \mathrm{~m} / \mathrm{s}$ ) are stronger than those in the other two carotids. The reflux area is also observed quite different for different carotids. Specifically, it can be observed that the reflux region is confined to a small area for the carotids $\mathrm{C} 1$ and $\mathrm{C} 3$, while it is filled over more than half of the area for the carotids C5 and C6. At the end of the systole (es) period, the magnitude of axial velocity decreases significantly so that the 


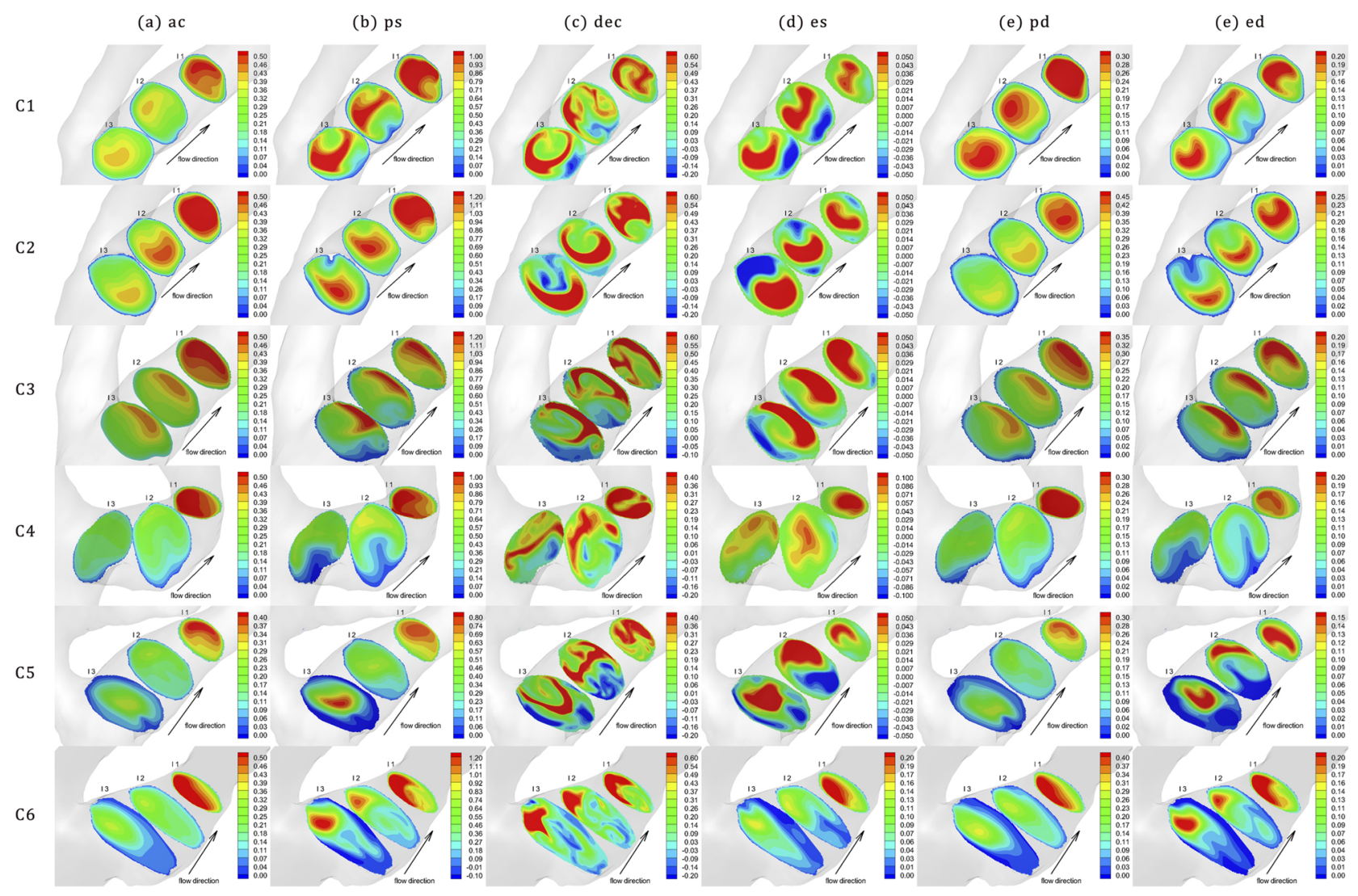

FIG. 4. The contours of the axial velocity on different cross sections in different carotids for (a)-(f) at ac, ps, dec, es, pd, and ed instants, respectively. Legend of contour: axial velocity $(\mathrm{m} / \mathrm{s})$.

magnitude of the positive and negative velocities becomes very close. For all the considered carotids at the es instant, the reversed flow is stronger in the region close to the blood vessel, which is different from those at the dec instant, when the reversed flow is also observed near the center of the blood vessel for the C2 and C6 carotids. It is also noticed that the reflux area at section I1 is negligible at both $\mathrm{dec}$ and es instants for all the six carotids. It is worth noting that the reflux area at the $\mathrm{I} 2$ section at the es instant varies significantly among different carotids, which can be more than $50 \%$ of the section area, e.g., C5 and C6, or less than 20\%, e.g., C4. Such differences are caused by the patient-specific carotid geometries and are different from what Jou and Berger ${ }^{40}$ found in their study that more than $30 \%$ of the area at the end of contraction (es) is occupied by the reflux area. The reflux area gradually disappears when the diastolic period is reached. At the pd instant, it is observed the overall patterns of the axial flow distributions are similar to those at the ps instant but with much smaller velocity magnitudes. At the ed instant, the reversed flows are also observed in some regions but not as intense as those at the es instant.

Here, we analyze the characteristics of secondary flow. The contours of the secondary flow magnitude in the sinus of different carotids are shown in Fig. 5. As seen, at the beginning of the acceleration phase (ac), the secondary flow speed is weak with its magnitude lower than $0.15 \mathrm{~m} / \mathrm{s}$ except for one carotid with large curvature, i.e., $\mathrm{C} 4$, in which the magnitude of the secondary flow is about $0.2 \mathrm{~m} / \mathrm{s}$. At the peak of the systole (ps) period, the magnitude of the secondary flow becomes comparable to the axial flow for all the carotids. The other common feature for the secondary flow of different carotids is that the region with strong secondary flow is mostly located in the region close to the wall except for carotid $\mathrm{C} 4$ at the $\mathrm{I} 3$ location. The maximum magnitudes of the secondary flows, however, are different for different carotids, which are higher for carotids C4 and C6 of a large curvature and a sudden decrease in the cross-sectional area, respectively. At the dec instant, the pattern of the secondary flow shares some common features with that at the ps instant but with more small scale structures and smaller portion of the cross section with strong secondary flows. At the es, fd, and ed instants, the secondary flow becomes significantly weaker than those at the ps and dec instants. It is interesting to notice that the overall patterns of the secondary flow at the ed instant are similar to those at the ps instant, e.g., carotid C4 and C6. It is worth noting that the magnitudes and the locations with strong secondary flows are different for different carotids for all the considered instants even though some overall similarities are observed. 
(a) ac

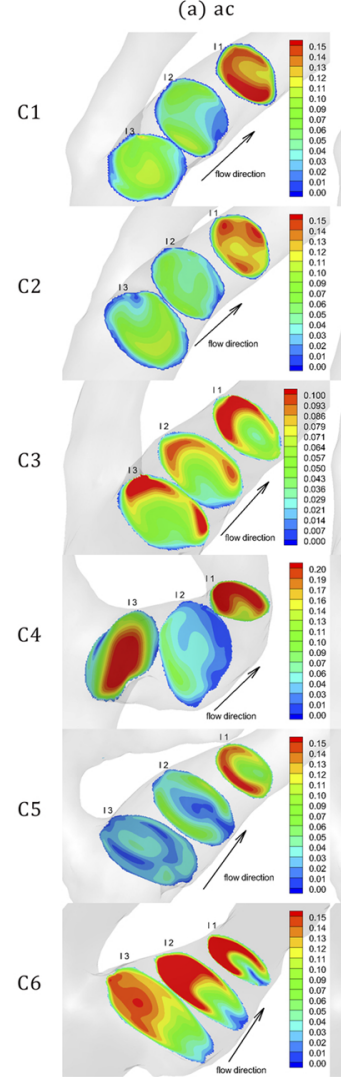

(b) p
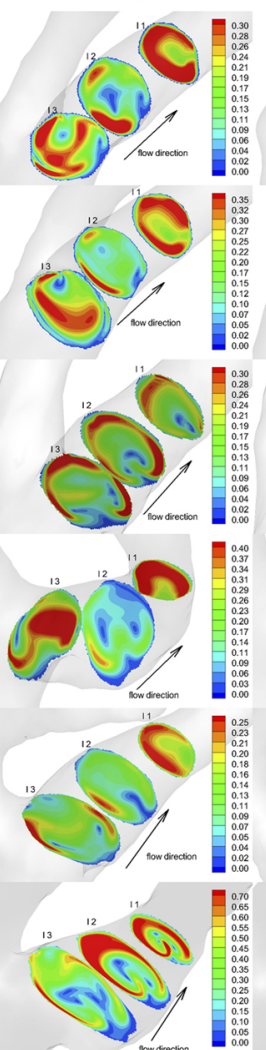

(c) dec
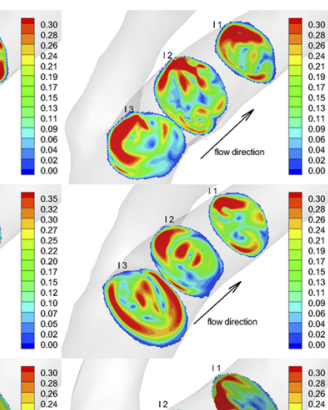

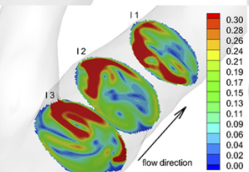

(d) es
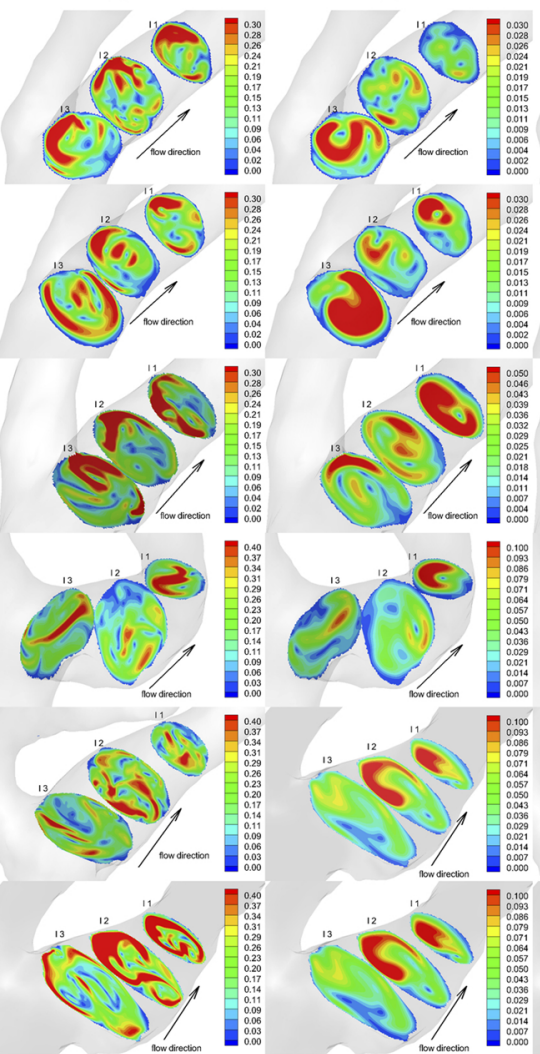

(e) pd

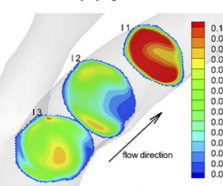

(f) ed
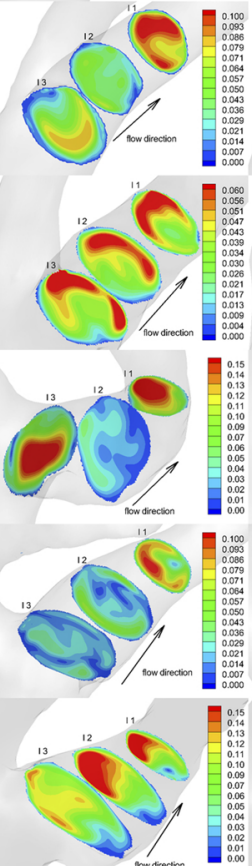
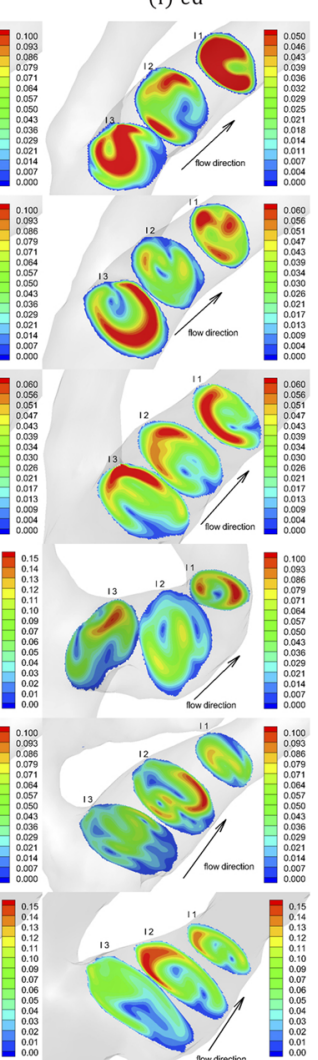

FIG. 5. The contours of the secondary flow magnitude on different cross sections in different carotids for (a)-(f) at ac, ps, dec, es, pd, and ed instants, respectively. Legend of contour: secondary flow magnitude $(\mathrm{m} / \mathrm{s})$.

We show in Fig. 6 the secondary flow ratio of different carotids to examine the dominance of the secondary flow during different phases for different carotids. In the acceleration stage (i.e., at ac instant), the value of SFR is small for the carotids C1, C2, and C3 but is large in some regions for the carotids $\mathrm{C} 4, \mathrm{C} 5$, and $\mathrm{C} 6$. When it reaches the deceleration phase (i.e., at dec instant) and the end of systole phase (i.e., at es instant), the secondary flow dominates over about $50 \%$ of the cross section (where SFR exceeds 0.8 ) for all the carotids except for $\mathrm{C} 1$.

Figures 7-12 show the streamline of the secondary flow at different cross sections of different carotids. In the acceleration phase (i.e., at ac instant), the patterns of streamlines are relatively simple flowing from one side of the blood vessel to the other side of the vessel except for carotids C4, C5, and C6, in which either a vortex is formed or the streamlines diverge from or converge to a point. At the peak of the systole phase (i.e., at the ps instant), we observe Deantype vortices (i.e., a pair of axial-wise vortices with the opposed rotational direction) in C3, C5, and C6, as showed by Rindt and van Steenhoven. ${ }^{19}$ The red region and blue region, which are colored by streamwise vorticity, represent the opposed rotational direction of the secondary flow at the cross sections. At the dec instant, the organized Dean-type vortices evolve into complicated coherent structures of different sizes and shapes. At the es instant, complex vortex structures still persist but with different shapes and locations. At the pd instant in the diastole phase, the patterns of streamlines become relatively simple with the overall pattern similar to those at the ac instant. At the ed instant, the streamlines have relatively simple patterns for the carotids $\mathrm{C} 1$ and $\mathrm{C} 2$, while they have coherent vortex pairs similar to those at the ps instant for some carotids, i.e., C4, C5, and C6.

The vortex structures are highly correlated with the flow state and the patient-specific geometry of the carotid. In Fig. 13, we show the vortex structures identified using the $\mathrm{Q}$ criterion for different carotids. As seen, no clear vortex structure exists at the ac instant during the acceleration phase for all carotids. At the ps instant, a pair of vortex tubes is observed except C3. This is consistent with the two long strip-shaped vortex structures that Cox et al. ${ }^{38}$ observed in curved blood vessels. The details of the vortex structures, however, are very different for different carotids. In carotid $\mathrm{C} 1$, the vortex tubes in the ICA and ECA are disconnected, which are connected in carotids $\mathrm{C} 3, \mathrm{C} 5$, and C6. At the dec instant, the vortex structures become fairly complex for all carotids as a result of the angle between the vortex tubes and the change in the incoming flow rate. At the es instant, tube-shaped vortex structures are 
(a) ac
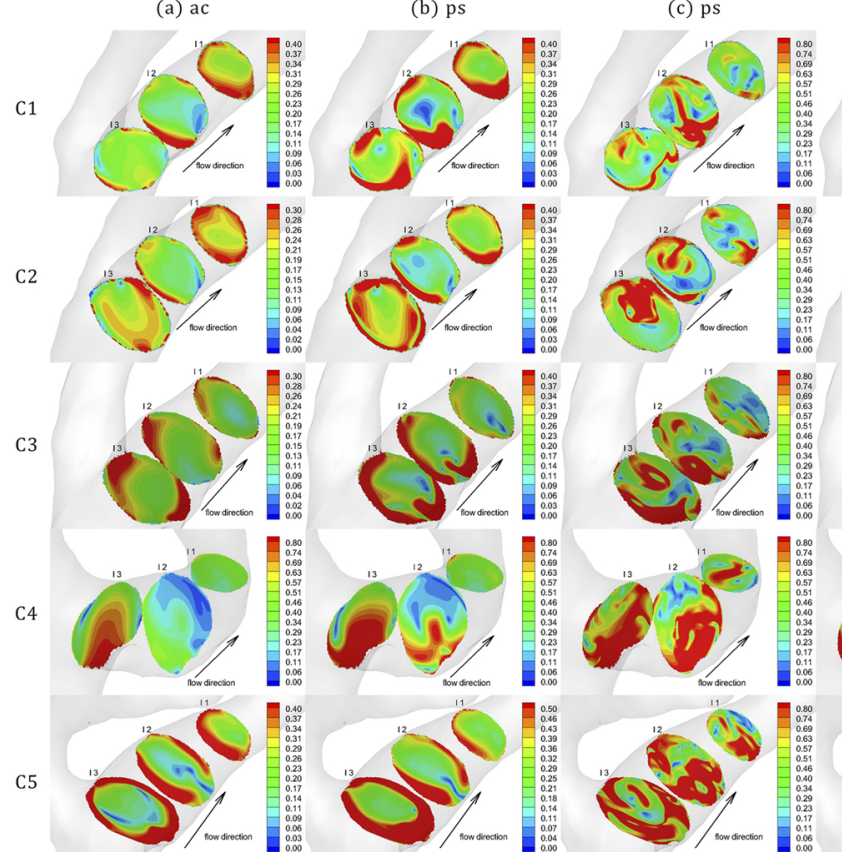

C5
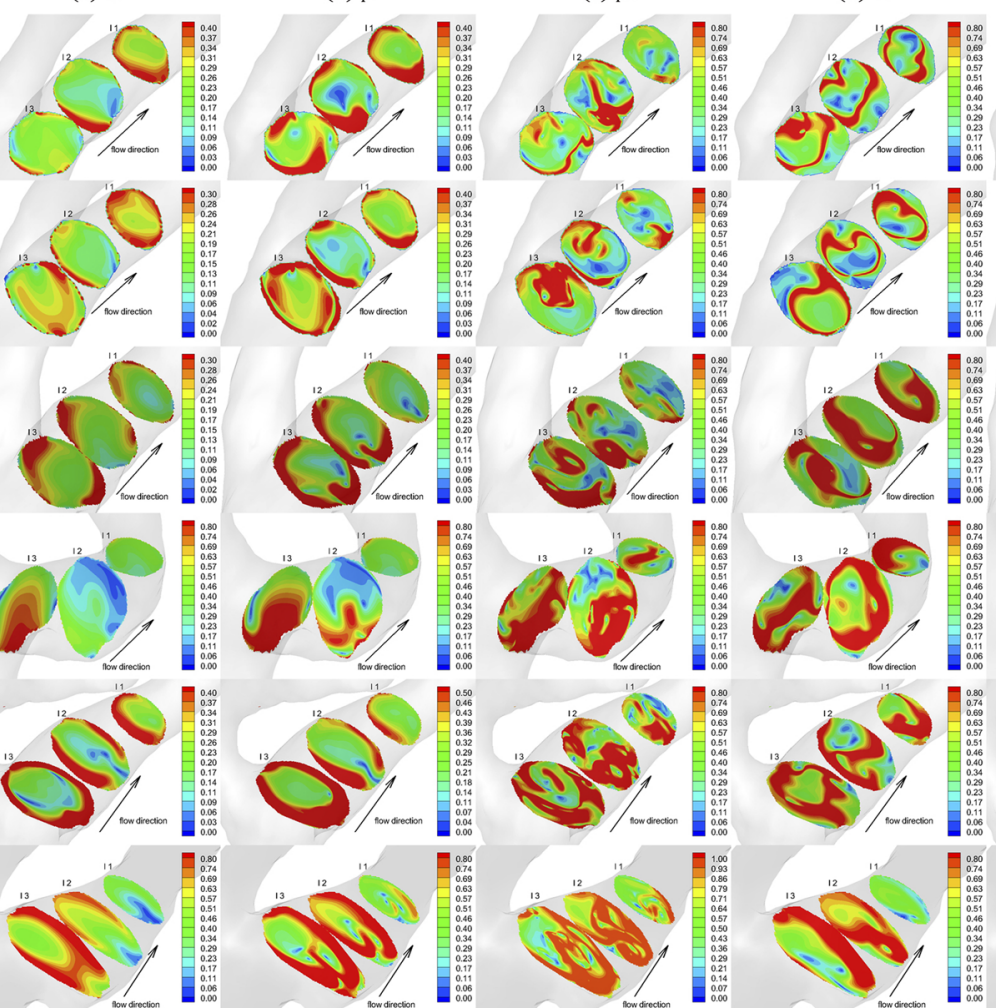
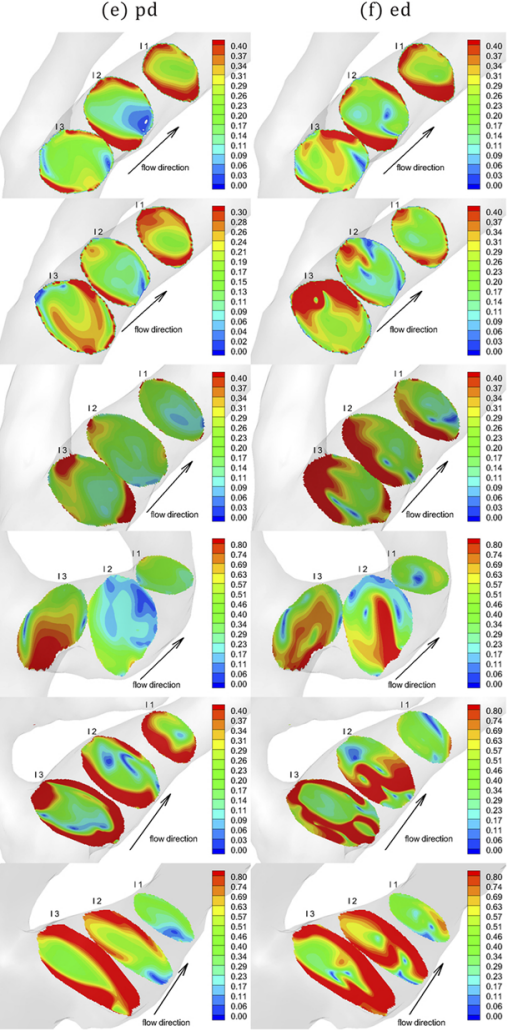

FIG. 6. The contours of the secondary flow ratio on different cross sections in different carotids for (a)-(f) at ac, ps, dec, es, pd, and ed instants, respectively.

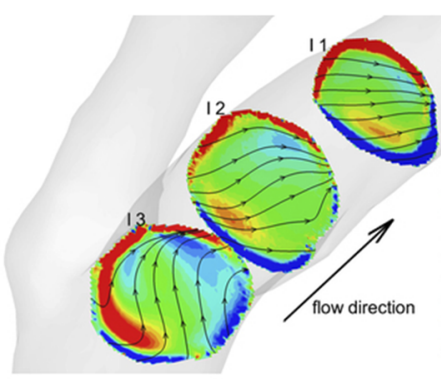

(a) $\mathrm{C} 1$

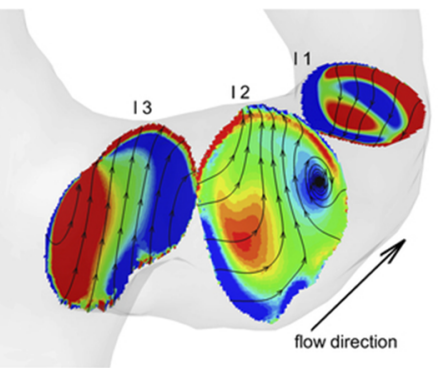

(d) $\mathrm{C} 4$
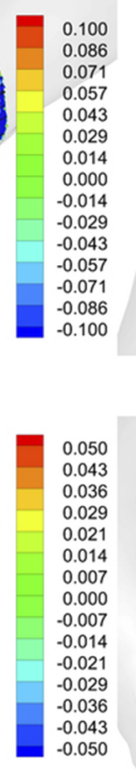

(low with th

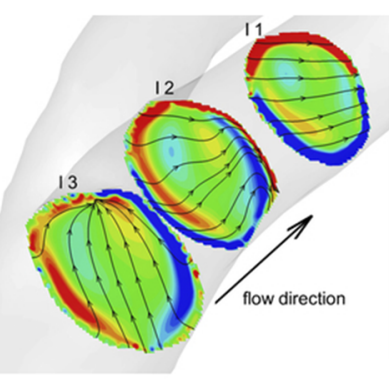

(b) $\mathrm{C} 2$

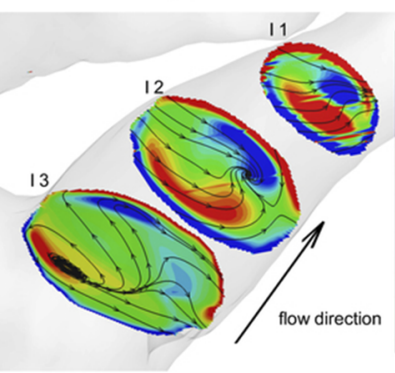

(e) $\mathrm{C5}$
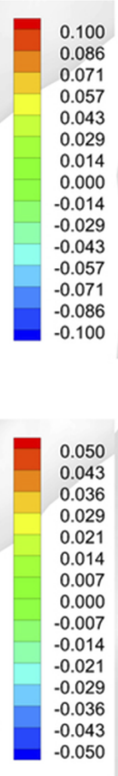

\begin{tabular}{|l|}
\hline 0.050 \\
\hline 0.043 \\
0.036 \\
0.029 \\
0.021 \\
0.014 \\
0.007 \\
0.000 \\
-0.007 \\
-0.014 \\
-0.021 \\
\hline 0.029 \\
\hline 0.036 \\
\hline 0.043 \\
\hline 0.050 \\
\hline
\end{tabular}

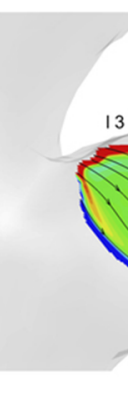

FIG. 7. Streamlines of the secondary flow with the contours of the streamwise vorticity on different cross sections in different carotids at the ac instant. 


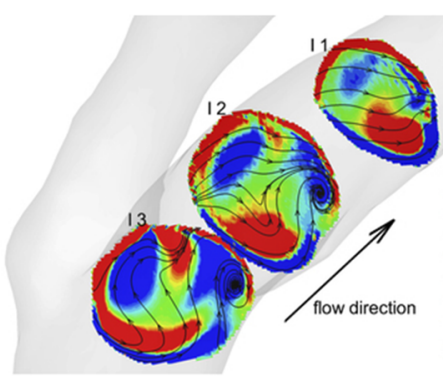

(a) $\mathrm{C} 1$

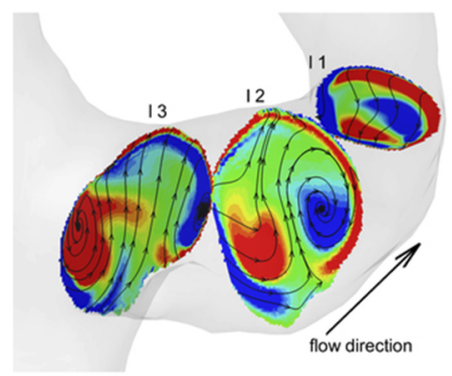

(d) $\mathrm{C} 4$
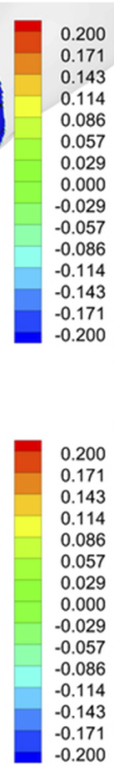

$-0.200$

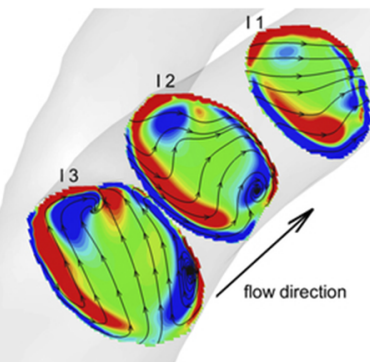

(b) $\mathrm{C2}$

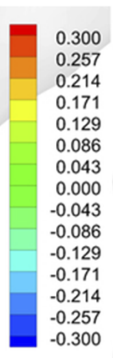

$-0.300$

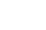

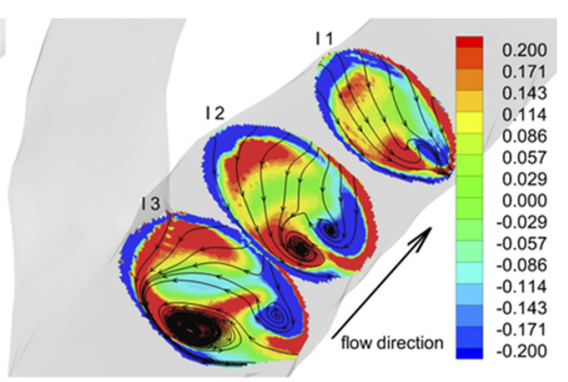

(c) $\mathrm{C} 3$

FIG. 8. Streamlines of the secondary flow with the contours of the streamwise vorticity on different cross sections in different carotids at the ps instant.

still observed for all carotids but less complicated than those in the dec instant. At the pd and ed instants, vortex structures can still be observed but less coherent than those in the ps, dec, and es stages.
It should be noticed that it is the complex carotid geometries that break the symmetry of the Dean-type vortices and cause the complicated spatial and temporal variations of the coherent vortex structure. As these vortices are strongly correlated with the

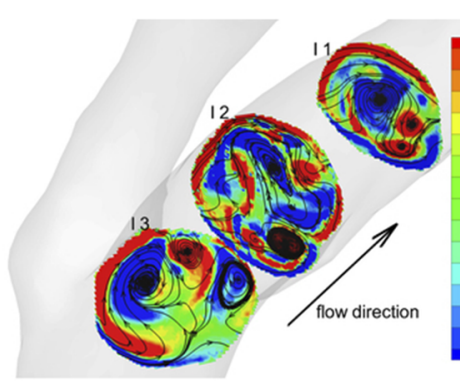

(a) $\mathrm{C} 1$

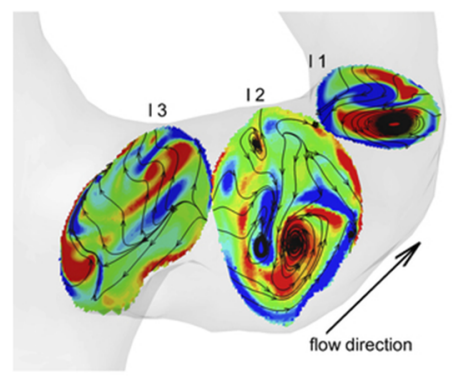

(d) $\mathrm{C} 4$
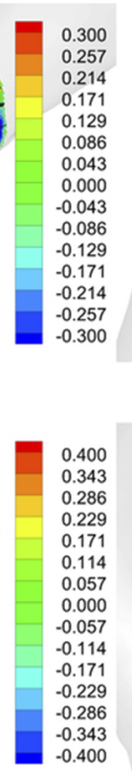

FIG. 9 . Streamlines of the secondary flow
101902 (2020); doi: $10.1063 / 5.0022092$
license by AIP Publishing

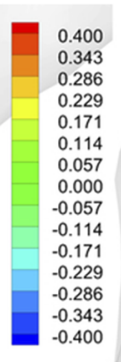

(b) $\mathrm{C} 2$

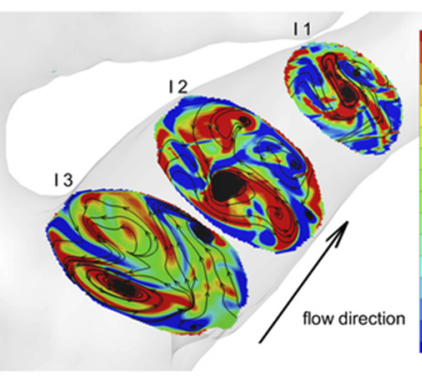

(e) $\mathrm{C5}$
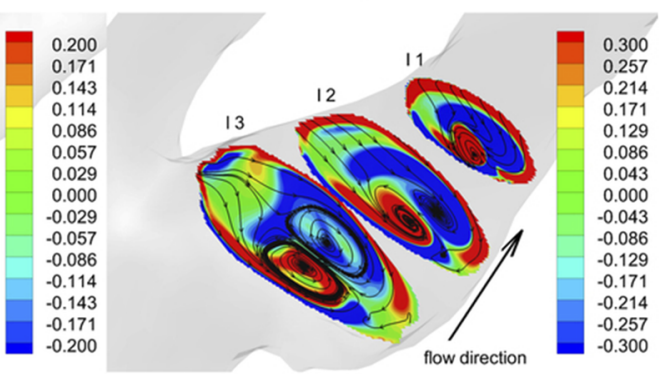

(f) $\mathrm{C} 6$

\section{.}




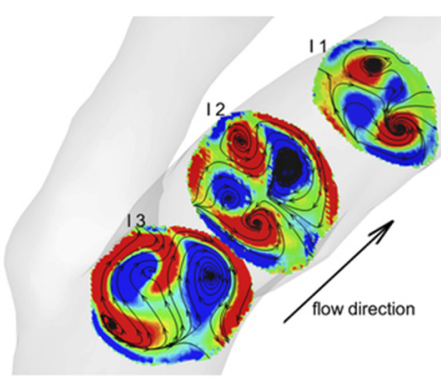

(a) $\mathrm{C} 1$

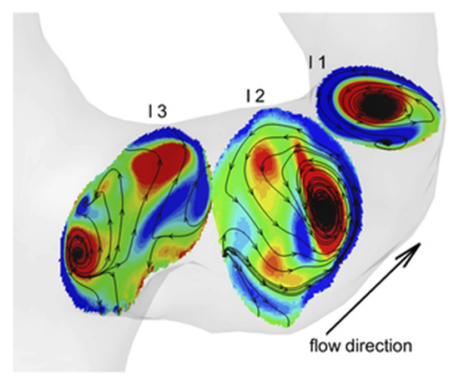

(d) $\mathrm{C} 4$

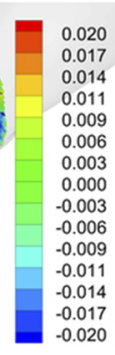

$-0.050$

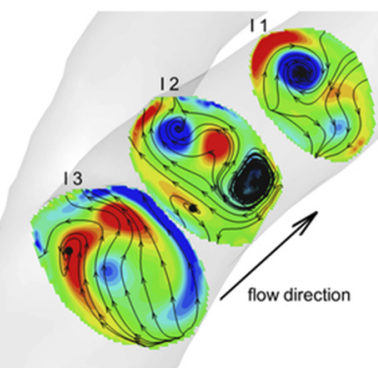

(b) $\mathrm{C} 2$

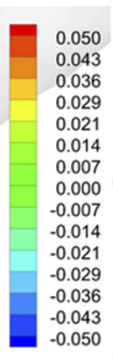

$-0.050$

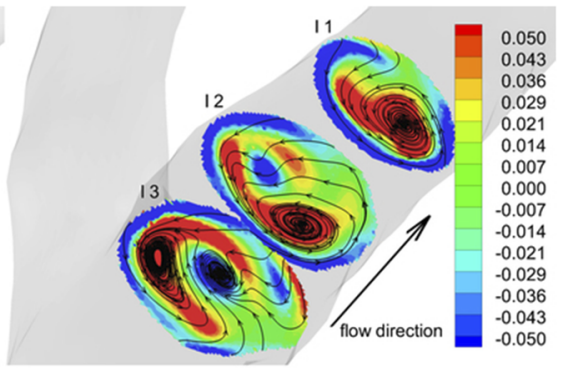

(c) $\mathrm{C} 3$

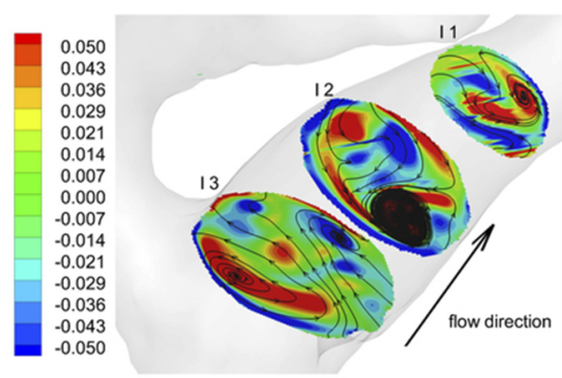

(e) $\mathrm{C5}$

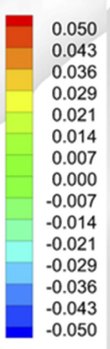

$-0.050$

FIG. 10. Streamlines of the secondary flow with the contours of the streamwise vorticity on different cross sections in different carotids at the es instant.

residence time of particles in the carotid sinus, a good understanding on their formation and evolution is important for discovering the mechanism for the formation of plaques. Because of the complex carotid geometries and the unsteadiness of the blood flow, it is difficult to quantitatively characterize the flow fields in the carotid sinus. In Sec. III B, we will define two types of variables for quantitative analysis of the flow in the carotid sinus.

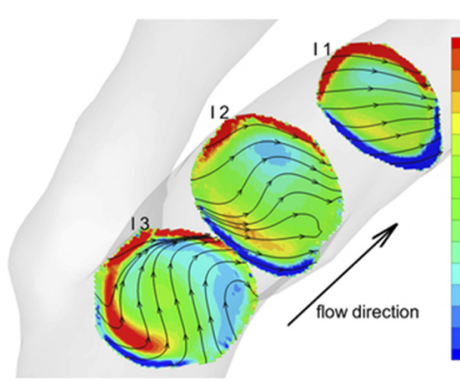

(a) $\mathrm{C} 1$

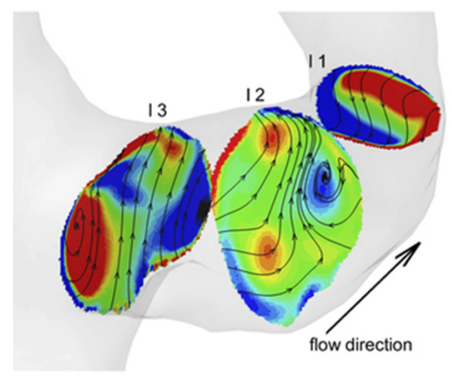

(d) $\mathrm{C} 4$
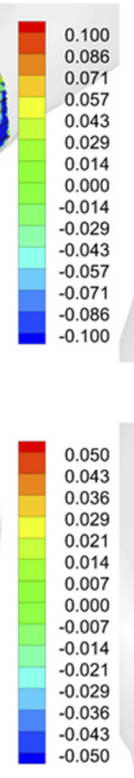

-0.043
-0.050 


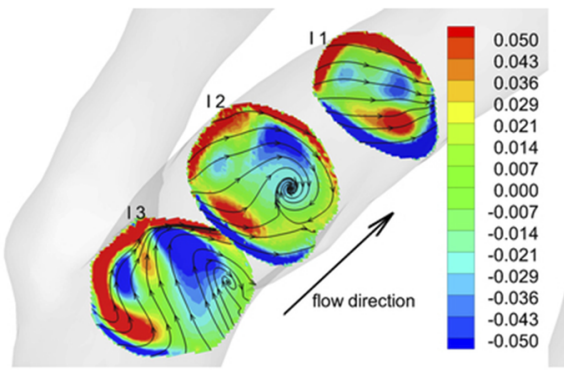

(a) $\mathrm{C} 1$

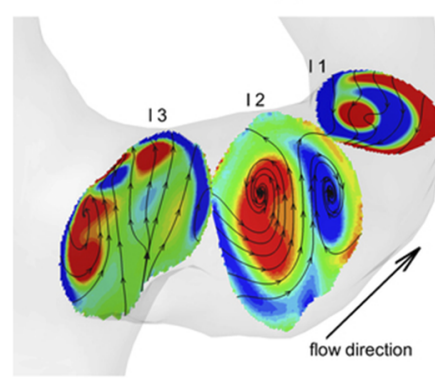

(d) $\mathrm{C} 4$

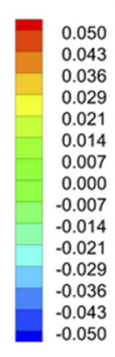

$-0.050$

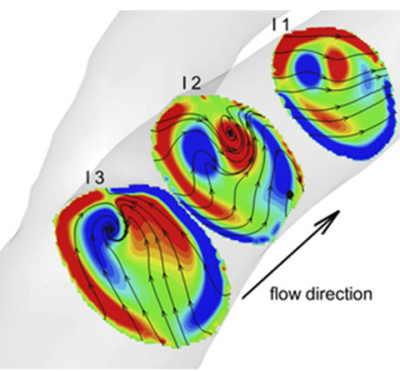

(b) $\mathrm{C} 2$
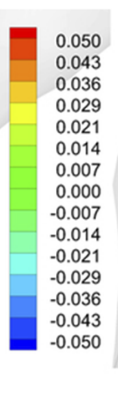

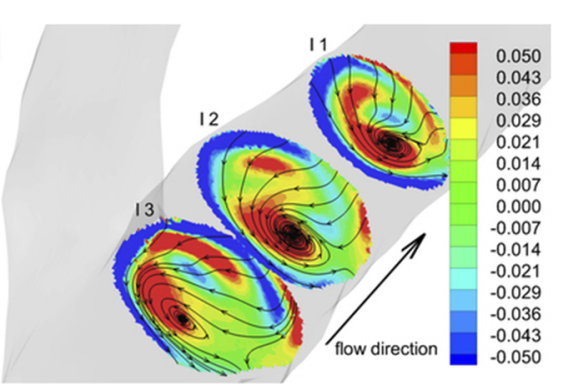

(c) $\mathrm{C} 3$

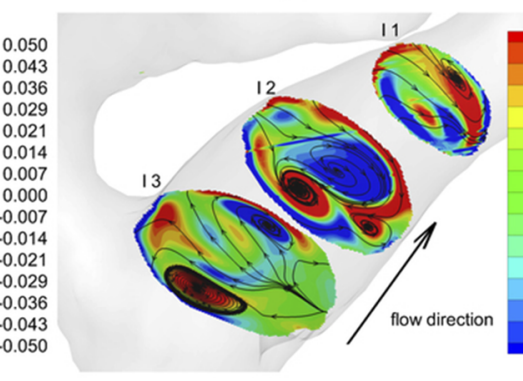

(e) $\mathrm{C5}$
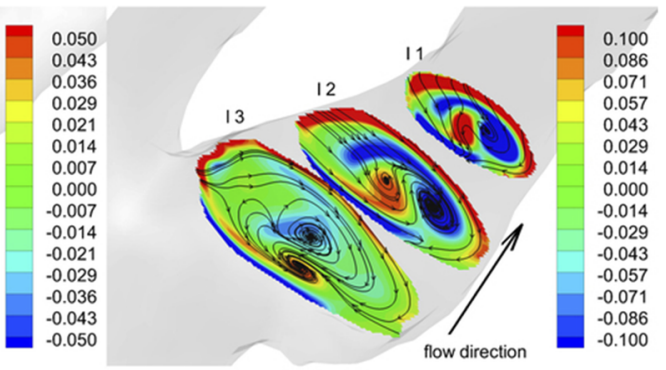

(f) $\mathrm{C} 6$

FIG. 12. Streamlines of the secondary flow with the contours of the streamwise vorticity on different cross sections in different carotids at the ed instant.

\section{B. Quantitative analysis of flow characteristics}

To analyze the axial velocity and the secondary flow quantitatively, three variables, i.e., the reversed flow ratio $(R F R)$, the standard deviation of the axial velocity fluctuations, $\sigma_{A V}$, and the standard deviation of the fluctuations of the velocity magnitude of the secondary flow, $\sigma_{S F}$, are defined as follows:

$$
R F R=\frac{A_{r e v}}{A},
$$

where $A_{\text {rev }}$ is the area of the cross section with reversed flow and $A$ is the total cross-sectional area,

$$
\sigma_{A V, S F}=\sqrt{\frac{1}{A} \int_{A}\left(u_{A V, S F}-\bar{u}_{A V, S F}\right)^{2} d A},
$$

where $u_{A V}$ is the axial velocity in the cross section, $u_{S F}$ is the velocity magnitude of the velocity of the secondary flow, and $\overline{(\cdot)}$ denotes averaging over the cross section.

Figure 14 shows the temporal variations of $R F R$ at the three cross sections for different carotids. At cross section I1 (close to the outlet of the carotid sinus), as shown in Fig. 6(a), the reversed flow is observed around $t=0.2 \mathrm{~s}$ (the dec instant) and $\mathrm{t}=0.4 \mathrm{~s}$ (the es instant). The values of RFR for all carotids, except for that from carotid C4, are close to 0.05 and 0.4 around the dec instant and the es instant, respectively. At cross sections I2 (in the middle of the carotid sinus) and I3 (close to the inlet of the carotid sinus), as shown in Figs. 6(b) and 6(c), similar temporal variations are observed with four peaks existing around $t=0.05 \mathrm{~s}, 0.25 \mathrm{~s}, 0.4 \mathrm{~s}$, and $0.6 \mathrm{~s}$ for all six carotids, which are corresponding to the troughs of the flow rate waveform. The maximum peak of the reversed flow ratio appears at $\mathrm{t}=0.4 \mathrm{~s}$ when the flow rate approaches to the minimum value (the es instant). At cross section $\mathrm{I} 3$, the peak values at $\mathrm{t}=0.4 \mathrm{~s}$ is about 0.4 for $\mathrm{C} 1$ and $\mathrm{C} 3,0.35$ for $\mathrm{C} 4,0.5$ for $\mathrm{C} 2$, and 0.6 for $\mathrm{C} 5$ and $\mathrm{C} 6$, respectively. For the other three peaks, the values of $R F R$ are all less than 0.4 for $\mathrm{C} 1-\mathrm{C} 4$, while they are close to 0.4 for C5 and C6. At section $\mathrm{I} 2$, the peak values of RFR are slightly smaller as compared those at the I3 section. It is worth noting that the $R F R$ values and total time with reversed flow at the I3 section are significantly larger for carotids C5 and C6 as compared with other carotids.

Figure 15 shows the temporal variations of $\sigma_{A V}$ and $\sigma_{S F}$ for all six carotids. We first examine the temporal variations of $\sigma_{A V}$. It is noticed that the waveforms of $\sigma_{A V}$ are close to the waveform of the flow rate except for section I1, where two peaks are observed near the ps instant. At section I1, as shown in Fig. 15(a), two peaks are observed before (acceleration phase) and after (deceleration phase) the peak of systole (ps instant), with a minimum value appearing around the ps instant for most carotids. At I2 and I3 sections, as shown in Figs. 15(b) and 15(c), respectively, the maximum standard deviation exists slightly after the ps instant, which is close to $0.8 \mathrm{~m} / \mathrm{s}$ for all carotids except for C6 (which is close to $1 \mathrm{~m} / \mathrm{s}$ at section I3). At sections I2 and I3, a second peak is also observed after the pd instant for all carotids, for which the value is $0.4 \mathrm{~m} / \mathrm{s}, 0.6 \mathrm{~m} / \mathrm{s}$, and $0.7 \mathrm{~m} / \mathrm{s}$ for $\mathrm{C} 1, \mathrm{C} 2$, and C5, respectively. We then examine the temporal variations of $\sigma_{S F}$ shown in Figs. 15(d)-15(f). It is observed that the temporal variations of $\sigma_{S F}$ are also strongly correlated with the inflow waveform as those observed in $\sigma_{A V}$. Similar with those of $\sigma_{A V}$, peaks are observed after the ps and pd instants for all carotids. At section I1, the positions of the second peak from different carotids are close to each other, i.e., immediately after the pd instant. At section $\mathrm{I} 2$, the second peak is not significant as those at sections I1 and I3 for 


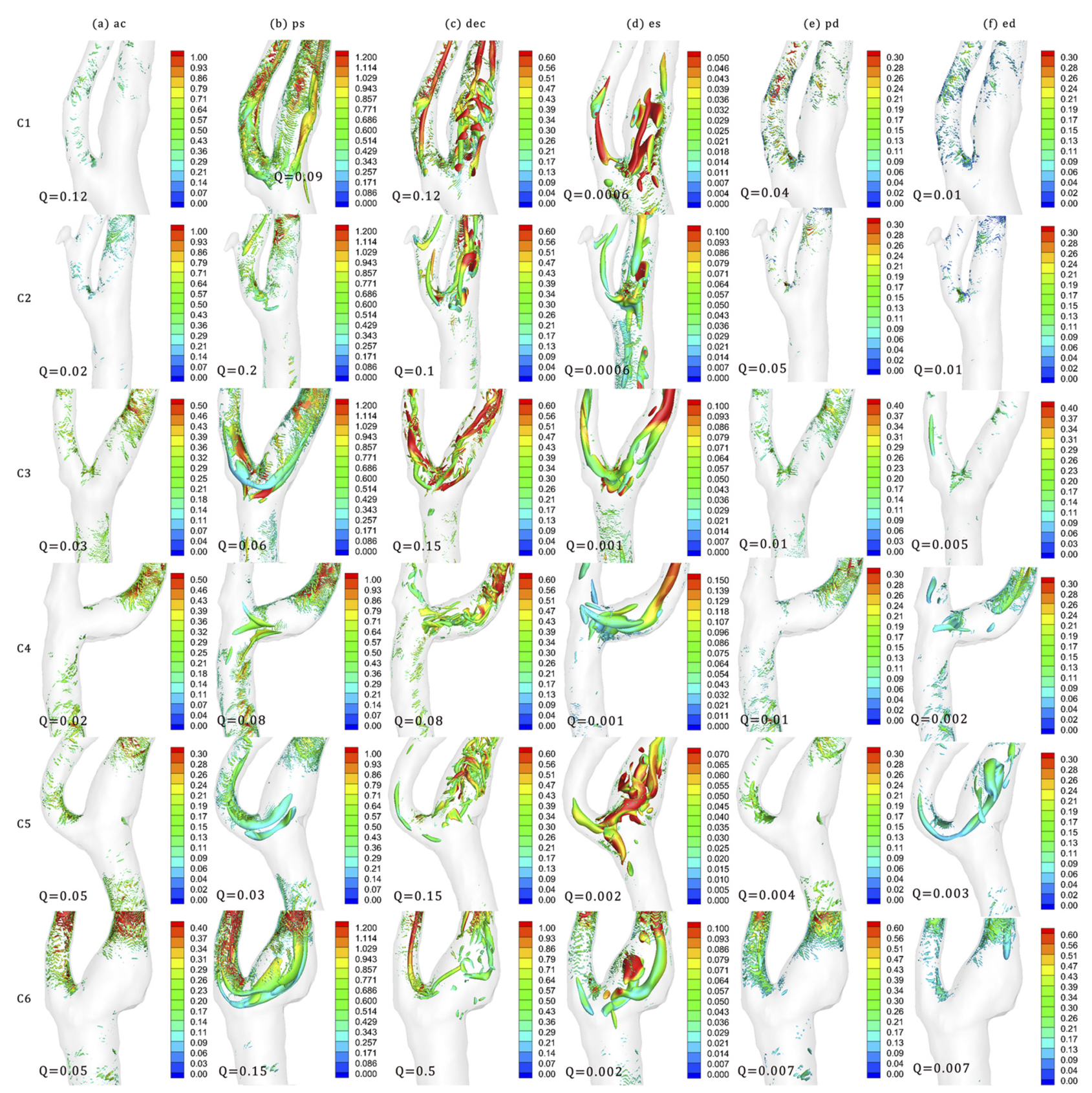

FIG. 13. The vortex iso-surfaces structures identified using the $Q$ criterion for (a)-(f) at ac, ps, dec, es, pd, and ed instants, respectively. Legend of contour: velocity magnitude $(\mathrm{m} / \mathrm{s})$.

most carotids except for carotids C5 and C6. It is also noticed that the values of $\sigma_{S F}$ from carotid C6 are significantly larger than other carotids for almost all the time at section I2. At section I3, considerable differences are observed for the positions of the second peak with that from the carotid C5 happening about $0.1 \mathrm{~s}$ after the pd instant.
In summary, we have observed similarities for different carotids in terms of the overall variations of $R F R, \sigma_{A V}$, and $\sigma_{S F}$ and their corresponding magnitudes. Meanwhile, we also observed differences between different carotids, such as the length of time with reversed flows and the magnitude of the second peak of $\sigma_{S F}$ and the time when it occurs. 


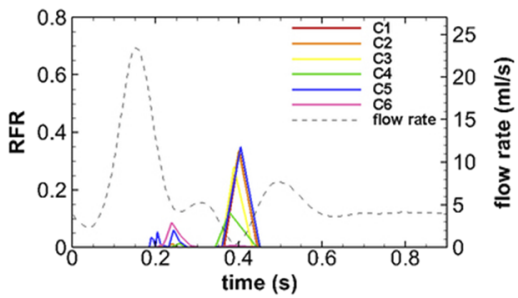

(a) I1

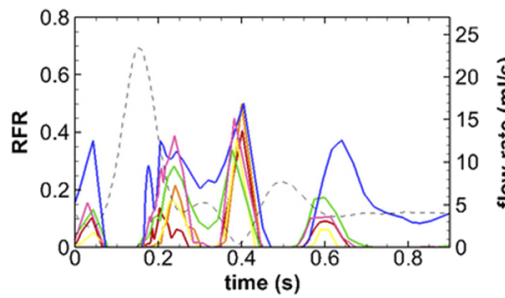

(b) 12

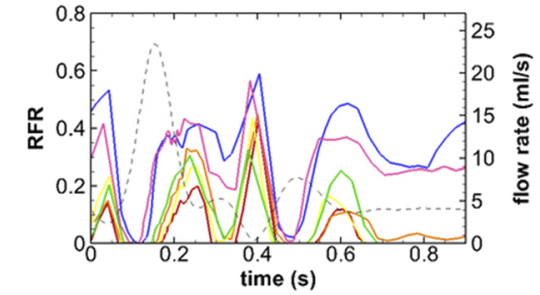

(c) 13

FIG. 14. The reversed flow ratio [RFR as defined in Eq. (5)] in one cardiac cycle for different carotids at different cross sections for (a) I1, (b) I2, and (c) I3, respectively. RFR defines the ratio of the area with reversed flow to the area of the cross section. This figure is to examine how RFR is correlated with the inflow waveform and the length of time with reversed flows for different carotids.

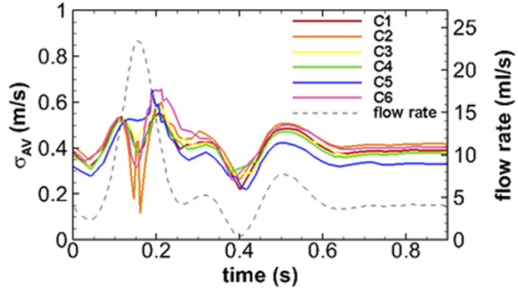

(a) I1

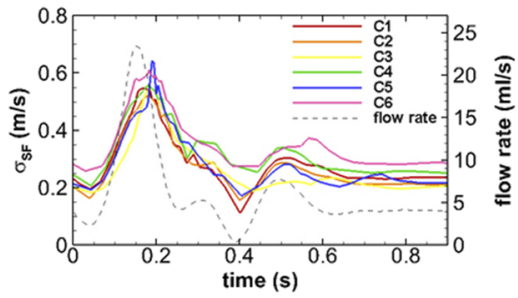

(d) I1

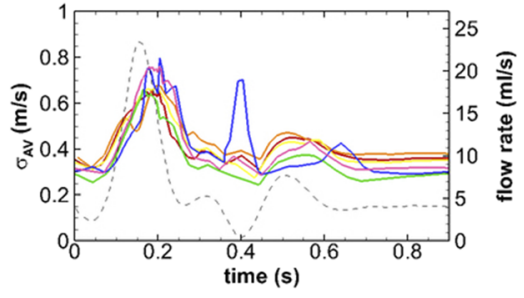

(b) I2

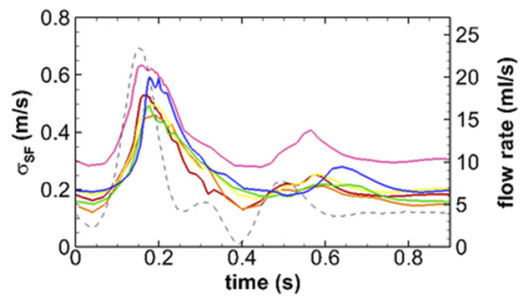

(e) 12

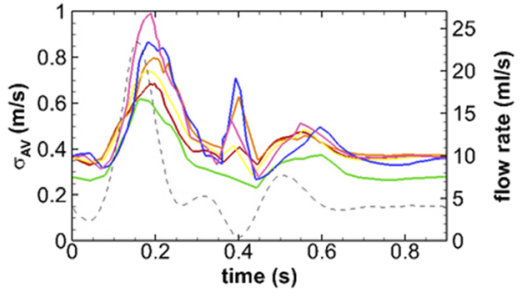

(c) 13

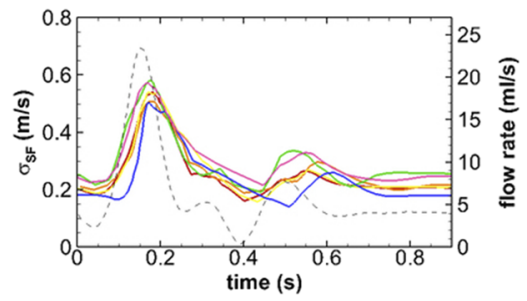

(f) I3

FIG. 15. The standard deviation of the fluctuations of the axial velocity $[(\mathrm{a})-(\mathrm{c})]$ and the secondary flow magnitude [(d)-(f)] in one cardiac cycle for different carotids at different cross sections for (a) and (d) I1, [(b) and (e)] I2, and [(c) and (f)] I3, respectively. The definition of $\sigma_{A V}$ and $\sigma_{S F}$ can be found in Eq. (6). The variables $\sigma_{A V}$ and $\sigma_{S F}$ show the degree of variation of the axial velocity and the secondary flow magnitude over the cross section. This figure is to examine how $\sigma_{A V}$ and $\sigma_{S F}$ are correlated with the inflow waveform and how they are influenced by carotid geometries.

\section{DISCUSSION}

Similarities are observed in the flow characteristics of different carotids, e.g., the existence of reversed flow, the overall pattern of the secondary flow, the key features of the coherent vortex structures, and the overall pattern of the temporal variations of $R F R, \sigma_{A V}$, and $\sigma_{S F}$. More importantly, differences caused by patient-specific carotid geometries are also observed regarding some key aspects of the flow characteristics. In this section, the relevance of the bulb width and the curvature of the carotid sinus with the differences observed in flow characteristics are discussed.

The bifurcation flare and the proximal curvature (defined in Fig. 1) are the two most important geometric factors affecting the early wall thickening in a carotid as pointed out by Bijari et al. ${ }^{28}$ They showed that the carotid with high flare and low curvature has the highest risk. That the carotid geometry can significantly affect the region with reversed flow and the secondary flows has also been reported in other studies. Bressloff ${ }^{41}$ demonstrated that the large bulb width can increase the area-weighted integral of the negative time-averaged shear stress. Lee et al..$^{35}$ showed that the curvature of the branch has a strong correlation with the disturbed flow. Pertold and Resch ${ }^{42}$ proposed that the small carotid sinus is beneficial for preventing the growth of atherosclerotic plaques. Nguyen et $a l^{43}$ examined the effects of the angles among ICA, ECA, and CCA on the wall shear stress for the ideal carotid geometry. Tada ${ }^{44}$ investigated the effect of bifurcation angle on axial velocity, wall shear stress, and oxygen transport for an ideal carotid model under steady inflow. A review on carotid geometry and atherosclerosis can be found in this paper. ${ }^{45}$ Our simulation results show that the carotids with high flare (C4, C5, and C6) have a larger region of reversed flows, as shown in Fig. 4, and are more dominated by secondary flows, as shown in Fig. 5. The length of time with reversed flows is also found to be longer for carotids C5 and C6 with high flare as compared with carotids (i.e., C1, C2, and C3) with low flare (as shown in Fig. 14). Based on the premise that longer time with reversed flow increases the residence time of the particle, this 
observation is supported by the clinical diagnosis carried out by Jiang et al., ${ }^{46}$ who found that the flare is associated with plaque vulnerability based on time-of-flight magnetic resonance angiography of 501 carotid arteries with nonstenotic atherosclerosis. One exception is carotid C4 for which the flare is high, but the length of time with reversed flow is similar to carotids $\mathrm{C} 1, \mathrm{C} 2$, and $\mathrm{C} 3$. This can be explained by the high proximal curvature of carotid $\mathrm{C} 4$, which can reduce the risk of getting atherosclerosis disease, as shown by Bijari et al. ${ }^{28}$ and is consistent with the study of 193 patients by Cui et al., ${ }^{47}$ which showed that symptomatic patients had increased the carotid bifurcation angle (i.e., low proximal curvature). From our simulations results, the curvature is also observed having a significant effect on the secondary flow and the coherent vortex structures. As shown in Fig. 5, the secondary flow in carotid $\mathrm{C} 2$ is not as dominant as that in carotids $\mathrm{C} 4, \mathrm{C} 5$, and $\mathrm{C} 6$ because of the small curvature of $\mathrm{C} 2$. In Fig. 13, it is observed that the flows in carotids $\mathrm{C} 4$ and $\mathrm{C} 5$ are featured by twisted vortex structures without long vortex tubes at the dec instant as compared with other carotids.

Flow structures in the carotid are significantly influenced by the bifurcation flare and the proximal curvature. Here, we attempt to categorize the patterns of the axial velocity (Fig. 4) for different carotids. In the work by Ford et al., ${ }^{48}$ the pattern of the axial velocity distribution is classified based on the shape of the region with high velocity magnitude. In this work, we classify the patterns of the axial velocity based on the axial variation of the region with high velocity magnitude. Specifically, we classify the flow patterns into four different categories, i.e., type SS-SL, type SS-DL, type DS-SL, and type DS-DL, as shown in Fig. 16, based on the similarities of the shape and locations of the region with high velocity magnitude. As seen in Fig. 4, the axial variation of the axial velocity can be classified as type I for most locations and instants (except for the dec instant) for carotids $\mathrm{C} 1, \mathrm{C} 2$, and C3, which have low flare. For carotids C4, C5, and C6, which have high flare, the patterns are quite different. For carotid C4, for which the curvature is high in addition to high flare, the axial velocity variation is of type I for cross sections I2 and I3, while it is of type IV for cross sections I1 and I2. For carotid C5, on the other hand, the axial variation is of type IV for both the variation between I 2 and I 3 and the variation between I1 and I2. For carotid C6, variations of type I are observed but with the shape close to a circle or half circle instead of the C-shape as observed in other carotids.

\section{CONCLUSION}

In this work, we simulated the blood flow in six human carotids using the sharp-interface curvilinear immersed boundary method. We examined the axial velocity, the secondary flow, and the vortex structure at different phases of the cardiac cycle and the temporal variations of the reversed flow ratio and the standard deviations of velocity fluctuations for different carotids. Both similarities and differences are observed among different carotids.

We first examined the patterns of the axial flows. It is observed that the axial flow is featured by fairly complex velocity distributions at the considered deceleration instant, while it is featured by relatively clear high speed and low speed regions at the other five considered instants for all the six carotids. Reversed flow is also observed for all carotids at the considered deceleration instants and the end of the systole. Differences on the axial flow are observed in terms of the maximum axial velocity, the shape, and locations of velocity patterns (such as the high speed and the low speed regions and the reversed flow region) and the starting time of different patterns (for instance, the reversed flow region in the C5 and C6 carotids is observed at the peak of the systole earlier than other carotids).

We then probed into the secondary flow, which is found to be dominant at the considered deceleration instant and the end of the systole for all the carotids. However, the relative size and the shape of the region dominated by the secondary flow are very different for different carotids. We also visualized the vortex structures. Similar features are observed for different carotids, such as two straight (or close to straight) vortex tubes at the peak of the systole and twisted vortex tubes with more small coherent structures at the deceleration instant and the end of the systole. Differences are observed regarding the details of vortex structures, e.g., the length and position of the vortex tubes.

Furthermore, we examined the length of the time with reversed flows via the reversed flow ratio RFR and the standard variations of the axial velocity and the secondary flow (i.e., $\sigma_{A V}$ and $\sigma_{S F}$ ) from different carotids. Similarities are observed in terms of the overall variations for most carotids. Differences are observed in terms of the magnitudes of $\sigma_{S F}$, the time when the second peak of $\sigma_{S F}$ occurs, and the length of time with high RFR. At last, we correlated the observed flow features with the flare and proximal curvature of the carotid, which have been used in the literature for

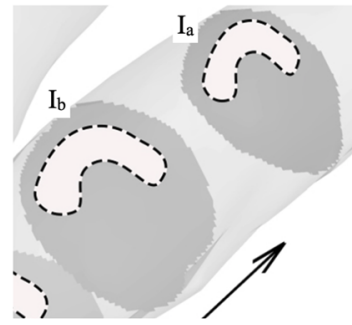

(a) Type SS-SL

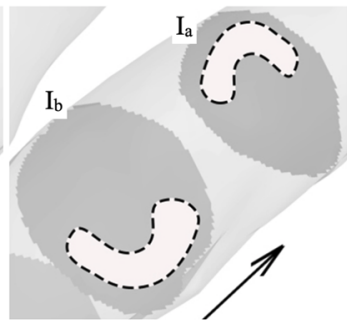

(b) Type SS-DL

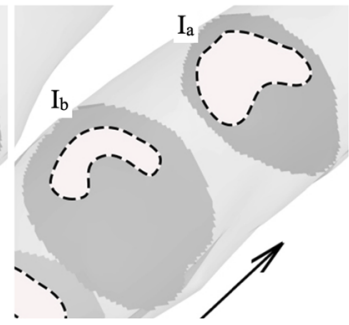

(c) Type DS-SL

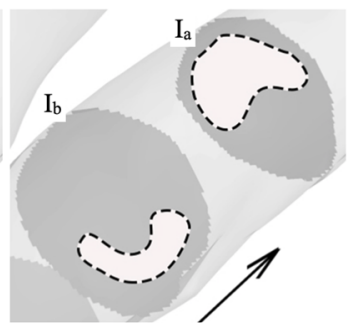

(d) Type DS-DL

FIG. 16. A schematic for four different axial variation types of the axial velocity patterns (as shown in Fig. 4), where $I_{a}$ and $I_{b}$ denote two adjacent cross sections. The four types are classified based on the shape and location of the region with high velocity magnitude (denoted by the light red region). 
evaluating the risk of different carotids getting atherosclerosis ${ }^{28}$ and are important factors affecting the formation of plaques, ${ }^{45}$ and found that the length of time with high RFR (which is related to the residence time of particles) and the intensity of velocity variations are higher for carotids C5 and C6 with the high flare and relatively low curvature.

At last, we attempted to classify the variation of the axial flow pattern. However, the flow structures in a patient-specific carotid are fairly complex and need to be investigated in a more rigorous way to examine their effects on the development of atherosclerosis. Specifically, turbulence-like flows are observed during the deceleration of the axial flow for patient-specific carotids, during which small geometrical imperfections may trigger the helical vortex pattern and the break down into turbulence as shown by Xu et al. ${ }^{49}$ and observed in this work. However, to the best of the authors' knowledge, no attention has been paid to how these turbulence-like flow structures affect the development of atherosclerosis, which needs to be investigated in future work.

This work suggests that the susceptibility to atherosclerosis is different for different people, and a patient-specific program is needed for the prevention and treatment of atherosclerosis. In this work, we only simulate the hemodynamics in different carotids. In order to make computer simulations play a better role in the prevention and treatment of atherosclerosis, computational models, which can predict the development of atherosclerosis in patient-specific carotids, need to be developed in future work. ${ }^{5,50,51}$

\section{ACKNOWLEDGMENTS}

This work was partially supported by the NSFC Basic Science Center Program for "Multiscale Problems in Nonlinear Mechanics" (Grant No. 11988102). (a)

(b)

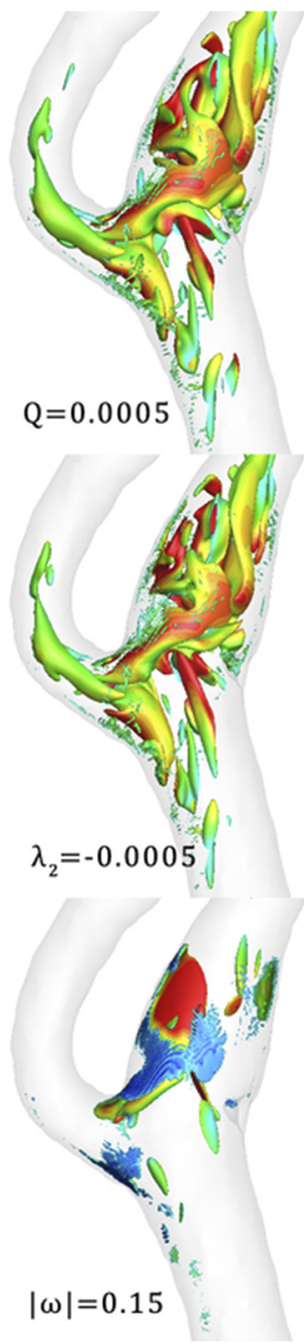

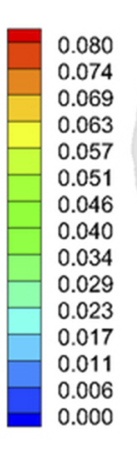
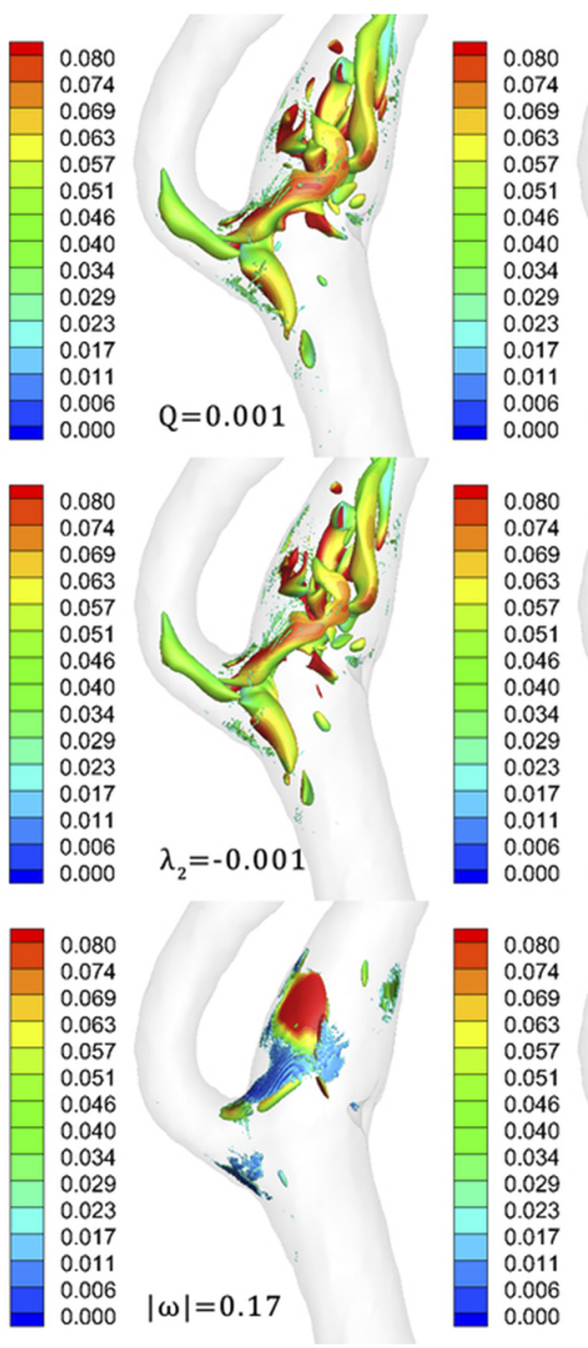

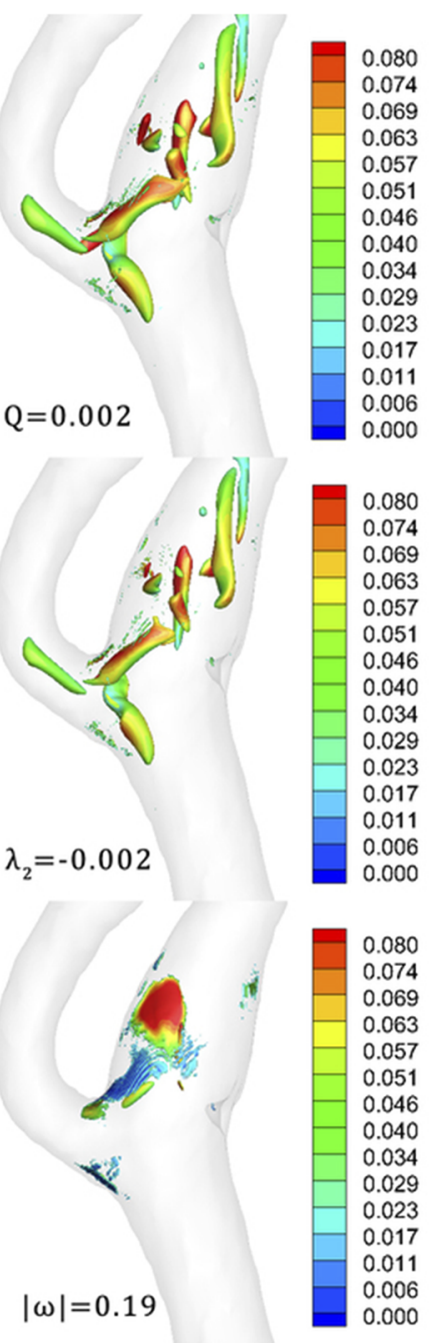

FIG. 17. Comparison of (a) $Q$ criterion, (b) $\lambda_{2}$ criterion, and (c) vorticity magnitude for identifying vortex structures in carotids (C5 at the es instant). The iso-surfaces are colored by velocity magnitude. 


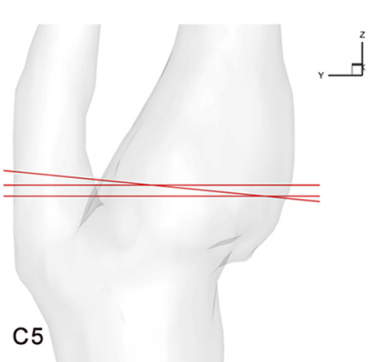

(a)

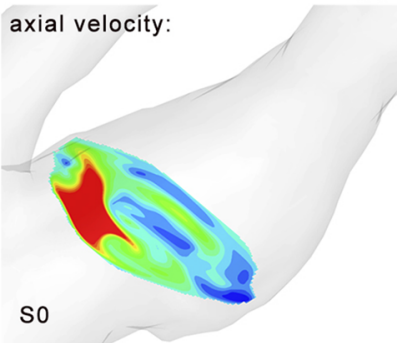

(c)

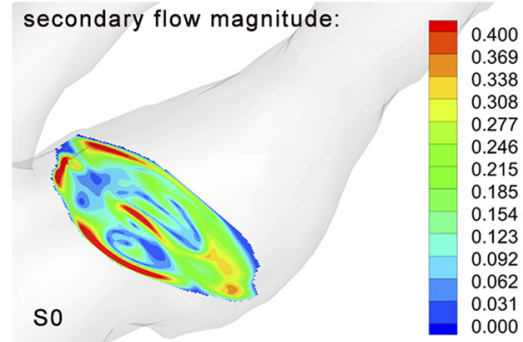

(f)
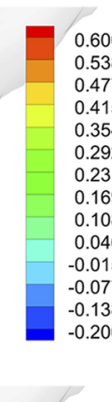

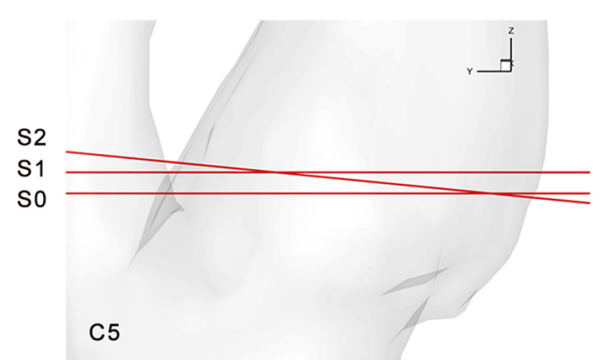

(b)

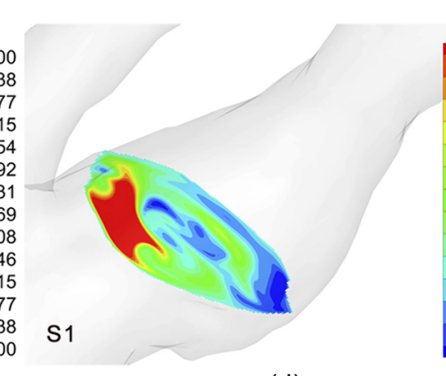

(d)

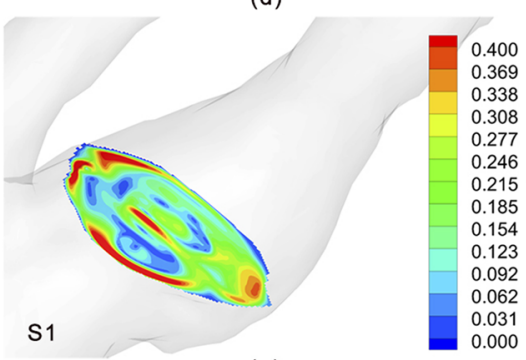

(g)

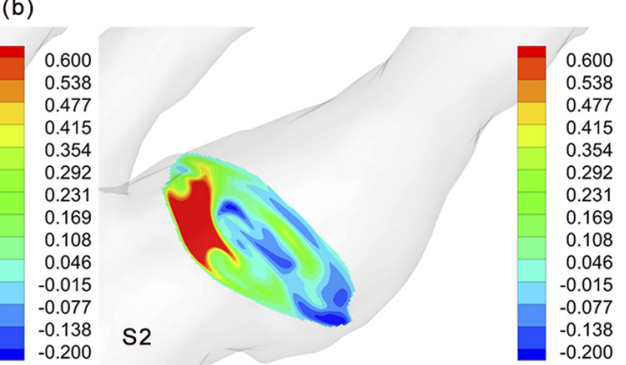

(e)

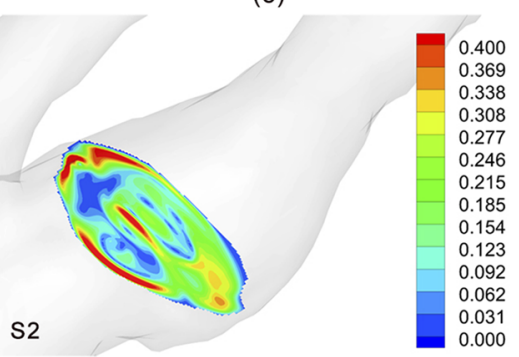

(h)

FIG. 18. Effects of slight changes in the orientation and location of the cross section on the observed flow structures for I3 from case C5 at the dec instant. (a) Selected cross section for testing; (b) enlarged view of (a); [(c)-(e)] contour of the axial velocity; and [(c)-(e)] contour of the secondary flow magnitude.

\section{APPENDIX A: TEST OF DIFFERENT VORTEX IDENTIFICATION METHODS}

Different vortex identification methods have been proposed in the literature, e.g., the method based on the Q criterion ${ }^{52}$ and the method based on the $\lambda_{2}$ criterion. ${ }^{53}$ Kohler et al. ${ }^{54}$ identified the vortex structure of the pulmonary arteries using the $\lambda_{2}$ criterion. Vedula et al. ${ }^{55}$ visualized the vortex structure in the left atrium using the $\lambda_{2}$ criterion. In this part, we examined the vortex structures identified using the vorticity magnitude, the Q criterion, and the $\lambda_{2}$ criterion. Figure 17(a) shows the iso-surfaces of the vorticity magnitude. It is seen that the vortex structures identified using the vorticity magnitude are mainly located in the near wall region where the magnitude of shear stress is high. Figures 17 (b) and 17 (c) show the vortex structures identified using the Q criterion and $\lambda_{2}$ criterion, respectively. As seen, the vortex structures identified using the two criteria are similar with each other. In this work, we arbitrarily choose the $\mathrm{Q}$ criterion for identifying the vortex structure in different carotids.

\section{APPENDIX B: FIOW FIELDS IN THE VICINITY OF THE SELECTED CROSS SECTION}

In this Appendix, we examine the differences by slightly changing the orientation of the location of the cross section, where we examine the flow fields. Specifically, we select C5 at the dec instant for carrying out this test, for which complex secondary flow is observed. As shown in Fig. 18(b), S0 is the cross section employed in this work, $\mathrm{S} 1$ is the cross section with a slight location change $(0.5 \mathrm{~mm})$ relative to $\mathrm{S} 0$, and $\mathrm{S} 2$ is the cross section with slight orientation changes $\left(5.6^{\circ}\right)$ relative to S0. As seen in Figs. 18(c)-18(e) and Figs. 18(f)-18(h) for the axial velocity and secondary flow magnitude, respectively, slight variations on the orientation and location of the cross sections do not change the observed flow structures significantly.

\section{DATA AVAILABILITY}

The flow field data and the carotid mesh data that support the findings of this study are available from X.Y. at 
xyang@imech.ac.cn upon reasonable request. The carotid DICOM files and the carotid mesh data are available from A.J.I. at andrew.iskander@wmchealth.org upon reasonable request. The authors are not aware of any additional permissions required to acquire the data.

\section{REFERENCES}

${ }^{1}$ D. N. Ku, D. P. Giddens, C. K. Zarins, and S. Glagov, "Pulsatile flow and atherosclerosis in the human carotid bifurcation. Positive correlation between plaque location and low oscillating shear stress," Arteriosclerosis 5, 293-302 (1985).

${ }^{2}$ C. Caro, J. Fitz-Gerald, and R. Schroter, "Atheroma and arterial wall shearobservation, correlation and proposal of a shear dependent mass transfer mechanism for atherogenesis," Proc. R. Soc. London, Ser. B 177, 109-133 (1971).

${ }^{3}$ D. L. Fry, "Acute vascular endothelial changes associated with increased blood velocity gradients," Circ. Res. 22, 165-197 (1968).

${ }^{4}$ M. H. Friedman, G. M. Hutchins, C. B. Bargeron, O. J. Deters, and F. F. Mark, "Correlation of human arterial morphology with hemodynamic measurements in arterial casts,” J. Biomech. Eng. 103, 204-207 (1981).

${ }^{5}$ J. M. Tarbell, Z.-D. Shi, J. Dunn, and H. Jo, "Fluid mechanics, arterial disease, and gene expression," Annu. Rev. Fluid Mech. 46, 591-614 (2014).

${ }^{6}$ V. Peiffer, S. J. Sherwin, and P. D. Weinberg, "Does low and oscillatory wall shear stress correlate spatially with early atherosclerosis? A systematic review," Cardiovasc. Res. 99, 242-250 (2013).

${ }^{7}$ V. Peiffer, E. M. Rowland, S. G. Cremers, P. D. Weinberg, and S. J. Sherwin, "Effect of aortic taper on patterns of blood flow and wall shear stress in rabbits: Association with age," Atherosclerosis 223, 114-121 (2012).

${ }^{8}$ A. R. Bond, S. Iftikhar, A. A. Bharath, and P. D. Weinberg, "Morphological evidence for a change in the pattern of aortic wall shear stress with age," Arterioscler., Thromb., Vasc. Biol. 31, 543-550 (2011).

${ }^{9}$ Z. Ding, K. Wang, J. Li, and X. Cong, "Flow field and oscillatory shear stress in a tuning-fork-shaped model of the average human carotid bifurcation," J. Biomech. 34, 1555-1562 (2001).

${ }^{10}$ V. Peiffer, S. J. Sherwin, and P. D. Weinberg, "Computation in the rabbit aorta of a new metric-The transverse wall shear stress-To quantify the multidirectional character of disturbed blood flow," J. Biomech. 46, 2651-2658 (2013).

${ }^{11}$ Y. Qiu and J. M. Tarbell, "Numerical simulation of pulsatile flow in a compliant curved tube model of a coronary artery," J. Biomech. Eng. 122, 77-85 (2000).

${ }^{12}$ P. B. Bijari, L. Antiga, D. Gallo, B. A. Wasserman, and D. A. Steinman, "Improved prediction of disturbed flow via hemodynamically-Inspired geometric variables," J. Biomech. 45, 1632-1637 (2012).

${ }^{13}$ S. Z. Zhao, X. Y. Xu, A. D. Hughes, S. A. Thom, A. V. Stanton, B. Ariff, and Q. Long, "Blood flow and vessel mechanics in a physiologically realistic model of a human carotid arterial bifurcation," J. Biomech. 33, 975-984 (2000).

${ }^{14}$ S.-W. Lee, L. Antiga, and D. A. Steinman, "Correlations among indicators of disturbed flow at the normal carotid bifurcation," J. Biomech. Eng. 131, 061013 (2009).

${ }^{15}$ R. L. T. Bevan, P. Nithiarasu, R. Van Loon, I. Sazonov, H. Luckraz, and A. Garnham, "Application of a locally conservative Galerkin (LCG) method for modelling blood flow through a patient-specific carotid bifurcation," Int. J. Numer. Methods Fluids 64, 1274-1295 (2010).

${ }^{16}$ S. Pinto, J. Campos, E. Azevedo, C. Castro, and L. Sousa, "Numerical study on the hemodynamics of patient-specific carotid bifurcation using a new mesh approach,” Int. J. Numer. Methods Biomed. Eng. 34, e2972 (2018).

${ }^{17}$ X. Yao, Z. Dai, X. Zhang, J. Gao, G. Xu, Y. Cai, and Z. Li, "Carotid geometry as a predictor of in-stent neointimal hyperplasia-A computational fluid dynamics study," Circ. J. 83, 1472 (2019).

${ }^{18} \mathrm{M}$. Nagargoje and R. Gupta, "Effect of sinus size and position on hemodynamics during pulsatile flow in a carotid artery bifurcation," Comput. Methods Prog. Biomed. 192, 105440 (2020).

${ }^{19}$ C. C. M. Rindt and A. A. van Steenhoven, "Unsteady flow in a rigid 3-D model of the carotid artery bifurcation," J. Biomech. Eng. 118, 90-96 (1996).
${ }^{20} \mathrm{M}$. W. Plesniak and K. V. Bulusu, "Morphology of secondary flows in a curved pipe with pulsatile inflow," J. Fluids Eng. 138, 101203 (2016).

${ }^{21}$ M. R. Najjari and M. W. Plesniak, "Evolution of vortical structures in a curved artery model with non-Newtonian blood-analog fluid under pulsatile inflow conditions," Exp. Fluids 57, 1-16 (2016).

${ }^{22}$ M. R. Najjari and M. W. Plesniak, "Secondary flow vortical structures in a $180^{\circ}$ elastic curved vessel with torsion under steady and pulsatile inflow conditions," Phys. Rev. Fluids 3, 013101 (2018).

${ }^{23}$ G. Janiga, "Quantitative assessment of $4 \mathrm{D}$ hemodynamics in cerebral aneurysms using proper orthogonal decomposition," J. Biomech. 82, 80-86 (2019).

${ }^{24}$ F.-B. Tian, L. Zhu, P.-W. Fok, and X.-Y. Lu, "Simulation of a pulsatile nonNewtonian flow past a stenosed 2D artery with atherosclerosis," Comput. Biol. Med. 43, 1098-1113 (2013).

${ }^{25}$ W. Choi, J. H. Park, H. Byeon, and S. J. Lee, "Flow characteristics around a deformable stenosis under pulsatile flow condition," Phys. Fluids 30, 011902 (2018).

${ }^{26}$ R. M. Lancellotti, C. Vergara, L. Valdettaro, S. Bose, and A. Quarteroni, "Large eddy simulations for blood fluid-dynamics in real stenotic carotids," Int. J. Numer. Methods Biomed. Eng. 33, e2868 (2017).

${ }^{27} \mathrm{X}$. Kang, W. Tang, and S. Liu, "Lattice Boltzmann method for simulating disturbed hemodynamic characteristics of blood flow in stenosed human carotid bifurcation," J. Fluids Eng. 138, 121104 (2016).

${ }^{28}$ P. B. Bijari, B. A. Wasserman, and D. A. Steinman, "Carotid bifurcation geometry is an independent predictor of early wall thickening at the carotid bulb," Stroke 45, 473-478 (2014).

${ }^{29}$ X. Yang, F. Sotiropoulos, R. J. Conzemius, J. N. Wachtler, and M. B. Strong, "Large-eddy simulation of turbulent flow past wind turbines/farms: The virtual wind simulator (VWiS)," Wind Energy 18, 2025-2045 (2015).

${ }^{30} \mathrm{~L}$. Ge and F. Sotiropoulos, "A numerical method for solving the 3D unsteady incompressible Navier-Stokes equations in curvilinear domains with complex immersed boundaries," J. Comput. Phys. 225, 1782-1809 (2007).

${ }^{31}$ I. Borazjani, L. Ge, and F. Sotiropoulos, "High-resolution fluid-structure interaction simulations of flow through a bi-leaflet mechanical heart valve in an anatomic aorta," Ann. Biomed. Eng. 38, 326-344 (2010).

${ }^{32}$ T. B. Le and F. Sotiropoulos, "Fluid-structure interaction of an aortic heart valve prosthesis driven by an animated anatomic left ventricle," J. Comput. Phys. 244, 41-62 (2013).

${ }^{33}$ A. Gilmanov, H. Stolarski, and F. Sotiropoulos, "Flow-structure interaction simulations of the aortic heart valve at physiologic conditions: The role of tissue constitutive model," J. Biomech. Eng. 140, 041003 (2018).

${ }^{34}$ A. J. Iskander, R. Naftalovich, and X. Yang, "The carotid sinus acts as a mechanotransducer of shear oscillation rather than a baroreceptor," Med. Hypotheses 134, 109441 (2020).

${ }^{35}$ S.-W. Lee, L. Antiga, J. D. Spence, and D. A. Steinman, "Geometry of the carotid bifurcation predicts its exposure to disturbed flow," Stroke 39, 2341-2347 (2008).

${ }^{36}$ F. N. Van de Vosse and N. Stergiopulos, "Pulse wave propagation in the arterial tree," Annu. Rev. Fluid Mech. 43, 467-499 (2011).

${ }^{37}$ D. W. Holdsworth, C. J. D. Norley, R. Frayne, D. A. Steinman, and B. K. Rutt, "Characterization of common carotid artery blood-flow waveforms in normal human subjects," Physiol. Meas. 20, 219-240 (1999).

${ }^{38}$ C. Cox, M. R. Najjari, and M. W. Plesniak, "Three-dimensional vortical structures and wall shear stress in a curved artery model," Phys. Fluids 31, 121903 (2019).

${ }^{39}$ M. D. Ford, N. Alperin, S. H. Lee, D. W. Holdsworth, and D. A. Steinman, "Characterization of volumetric flow rate waveforms in the normal internal carotid and vertebral arteries," Physiol. Meas. 26, 477 (2005).

${ }^{40}$ L.-D. Jou and S. A. Berger, "Numerical simulation of the flow in the carotid bifurcation," Theor. Comput. Fluid Dyn. 10, 239-248 (1998).

${ }^{41} \mathrm{~N}$. W. Bressloff, "Parametric geometry exploration of the human carotid artery bifurcation," J. Biomech. 40, 2483-2491 (2007).

${ }^{42} \mathrm{~K}$. Perktold and M. Resch, "Numerical flow studies in human carotid artery bifurcations: Basic discussion of the geometric factor in atherogenesis," J. Biomed. Eng. 12, 111-123 (1990). 
${ }^{43}$ K. T. Nguyen, C. D. Clark, T. J. Chancellor, and D. V. Papavassiliou, "Carotid geometry effects on blood flow and on risk for vascular disease," J. Biomech. 41, $11-19$ (2008)

${ }^{44} \mathrm{~S}$. Tada, "Computational study of the influence of bifurcation angle on haemodynamics and oxygen transport in the carotid bifurcation," Biomed. Eng.: Appl., Basis Commun.31, 1950024 (2019).

${ }^{45}$ K. Spanos, G. Petrocheilou, C. Karathanos, N. Labropoulos, D. Mikhailidis, and A. Giannoukas, "Carotid bifurcation geometry and atherosclerosis," Angiology 68, 757-764 (2017).

${ }^{46}$ P. Jiang, Z. Chen, D. S. Hippe, H. Watase, B. Sun, R. Lin, Z. Yang, Y. Xue, $\mathrm{X}$. Zhao, and C. Yuan, "Association between carotid bifurcation geometry and atherosclerotic plaque vulnerability: A Chinese atherosclerosis risk evaluation study," Arterioscler., Thromb., Vasc. Biol. 40, 1383-1391 (2020).

${ }^{47}$ Y. Cui, X. Lv, F. Wang, J. Kong, H. Zhao, Z. Ye, C. Si, L. Pan, P. Liu, and J. Wen, "Geometry of the carotid artery and its association with pathologic changes in a Chinese population," Front. Physiol. 10, 1628 (2020).

${ }^{48}$ M. D. Ford, Y. J. Xie, B. A. Wasserman, and D. A. Steinman, "Is flow in the common carotid artery fully developed?"” Physiol. Meas. 29, 1335 (2008).
${ }^{49}$ D. Xu, A. Varshney, X. Ma, B. Song, M. Riedl, M. Avila, and B. Hof, "Nonlinear hydrodynamic instability and turbulence in pulsatile flow," Proc. Natl. Acad. Sci. U. S. A. 117, 11233-11239 (2020).

${ }^{50}$ S. Kenjereš and A. de Loor, "Modelling and simulation of low-density lipoprotein transport through multi-layered wall of an anatomically realistic carotid artery bifurcation," J. R. Soc., Interface 11, 20130941 (2014).

${ }^{51}$ A. C. Stamou and J. M. Buick, "An LBM based model for initial stenosis development in the carotid artery," J. Phys. A: Math. Theor. 49, 195602 (2016).

${ }^{52}$ J. C. R. Hunt, A. A. Wray, and P. Moin, "Eddies, streams, and convergence zones in turbulent flows," in Proceedings of the Summer Program 1988 (Center for Turbulence Research, 1988), N89-24555.

${ }^{53}$ J. Jeong and F. Hussain, "On the identification of a vortex," J. Fluid Mech. 285, 69-94 (1995).

${ }^{54}$ B. Kohler, R. Gasteiger, U. Preim, H. Theisel, M. Gutberlet, and B. Preim, “Semiautomatic vortex extraction in 4D PC-MRI cardiac blood flow data using line predicates," IEEE Trans. Visualization Comput. Graphics 19, 2773-2782 (2013).

${ }^{55} \mathrm{~V}$. Vedula, R. George, L. Younes, and R. Mittal, "Hemodynamics in the left atrium and its effect on ventricular flow patterns," J. Biomech. Eng. 137, 111003 (2015). 